\title{
Upper Mantle Radial Anisotropy Under the Indian Ocean from Higher Mode Surface Waves and a
} Hierarchical Transdismensional Approach

\author{
Erik Weidner ${ }^{1}$, Caroline Beghein ${ }^{1}$, Q. Huang ${ }^{2}$, N. Schmerr ${ }^{2}$ \\ ${ }^{1}$ Department of Earth, Planetary, and Space Sciences, University of California Los Angeles, Los Angeles, CA, USA \\ ${ }^{2}$ Department of Geology, University of Maryland, College Park, MD, USA
}

This article is a non-peer reviewed preprint submitted to EarthArXiv. It has also been submitted to Geophysical Journal International.

\section{SUMMARY}

We investigated the likelihood of radial anisotropy in the shallow and deep upper mantle, including the mantle transition zone (MTZ) under the Indian Ocean. Seismic anisotropy can be an indicator of mantle deformation through lattice preferred orientation of anisotropic crystals in the mantle. It has thus the potential to illuminate Earth's dynamic interior, but previous seismic tomography studies have not achieved consensus on the existence of radial anisotropy below $\sim 250 \mathrm{~km}$ depth. We developed a fully non-linear transdimensional hierarchical Bayesian Markov Chain Monte Carlo approach to invert fundamental and higher mode surface wave dispersion data and applied it to a subset of a global Love and Rayleigh wave dataset. We obtained posterior model parameter distributions for shear-wave velocity $\left(V_{S}\right)$ and radial anisotropy $\xi$ under the Indian Ocean. These posterior model distributions were used to calculate the probability of having radial anisotropy at different depths. We demonstrated that separate inversions of Love and Rayleigh waves yield 
models compatible with the results of joint inversions within uncertainties. The obtained pattern of $V_{S}$ anomalies agrees with previous studies. They display negative anomalies along ridges in the uppermost mantle, but those are stronger than for regularized inversions. The Central Indian Ridge and the Southeastern Indian Ridge present velocity anomalies that extend to $\sim 200 \mathrm{~km}$ depth whereas the Southwestern Indian Ridge seems to have a shallower origin. Weaker, laterally variable velocity perturbations were found at larger depths. The anisotropy models differ more strongly from regularized inversion results, especially below $100 \mathrm{~km}$ depth. Apart from a fast horizontally polarized shear-wave signal in the top $100 \mathrm{~km}$, likely reflecting the horizontal plate motion due to asthenospheric deformation, no clear relation to surface geology was detected in the anisotropy models. We found that, although the anisotropy model uncertainties are rather large, and lateral variations are present, the data generally prefer at least $1 \%$ anisotropy in the MTZ with fast vertically polarized shear waves, within errors. Incorporating group velocity data did not help better constrain deep structure by reducing parameter trade-offs. We also tested the effect of prior constraints on the 410- and 660-km topography and found that the undulations of these discontinuities had little effect on the resulting models.

Key words: Seismic tomography - Seismic anisotropy - Surface waves and free oscillations - Inverse theory - Probability distributions

\section{INTRODUCTION}

Seismic anisotropy, which is the directional dependence of wave propagation, can provide clues to the properties and dynamics of the mantle. Radial anisotropy is the change in seismic wave velocity between the horizontal and vertical direction. Seismic anisotropy can occur via shape preferred orientation (SPO), which occurs where multiple isotropic mediums are stacked on top of each other, such as melt pockets and cracks (Kendall \& Silver 1998; Faccenda et al. 2008; Sakamaki et al. 2013). Although the individual mediums are isotropic, the bulk medium has directional dependence. Seismic anisotropy can also develop due to the crystallographic or lattice preferred orientation (CPO or LPO) of intrinsically anisotropic crystals. If such 
crystals can deform by dislocation creep, they may partially align within the strain field caused by mantle convection, resulting in bulk anisotropy that may be observable at the seismic scale. There are abundant observations of seismic anisotropy in the crust and upper mantle, both globally and regionally. Radial anisotropy was first proposed by Anderson (1962) to explain apparent discrepancies between shear-wave velocity models derived from inversions of Love wave dispersion curves and those resulting from Rayleigh wave data. The Preliminary Reference Earth Model (PREM) (Dziewonski \& Anderson 1981) was the first global model to include this type of anisotropy in the top $220 \mathrm{~km}$ of the mantle. Since then, many global and regional studies have found evidence for its presence in the uppermost mantle (Montagner \& Tanimoto 1991; Shapiro \& Ritzwoller 2002; Visser et al. 2008a).

LPO of olivine is the prevailing explanation for the existence of seismic anisotropy at these depths due to the abundance of this mineral in the mantle and its high single crystal anisotropy (Mainprice et al. 2005). Mantle convection is thought to be the origin of LPO in the upper mantle, which is supported by observations of the seismically fast axes in the asthenosphere aligning with absolute plate motion (Becker et al. 2003; Burgos et al. 2014; Debayle \& Ricard 2013; Beghein et al. 2014). Therefore, improving our constraints on seismic anisotropy is important to better understand the nature of mantle convection. In particular, determining whether it is present in the mantle transition zone (MTZ) is important since this region likely plays an important role in Earth's thermal evolution, but it is a challenging problem. Improving constraints on upper mantle anisotropy can also help determine the depth of the lithosphere-asthenosphere boundary (LAB), which directly relates to the fundamental concept of plate tectonics. Additionally, vertical changes in seismic anisotropy can indicate layering in the upper mantle. This, in turn, can provide insight on the LAB depth and can indicate the existence of compositional boundaries (Smith et al. 2004; Yuan \& Romanowicz 2010; Beghein et al. 2014).

While the presence of seismic anisotropy in the upper $250 \mathrm{~km}$ of the mantle, in the lowermost mantle, and in the inner core is well established, its existence in the rest of the mantle is still debated. For decades, seismic anisotropy had not been observed in the deep upper mantle or in most of the lower mantle, which was interpreted as an indication that deformation at those depths did not produce LPO (Karato \& Li 1992). More recently, however, radial and azimuthal anisotropy observations below $250 \mathrm{~km}$ depth, including in and below the MTZ, have challenged this view (e.g. Gung et al. 2003a; Panning \& Romanowicz 2006a; Yuan \& Beghein 2013, 2014; Lynner \& Long 2015; Huang et al. 2019). Constraining seismic anisotropy in the deep upper mantle is, however, not straightforward. Body waves do not generally have good 
vertical resolution as they travel quasi vertically. Some shear-wave splitting analyses using source-side measurements have been able to identify seismic anisotropy originating from large depths, though they cannot separate the contribution of the uppermost mantle from that of the transition zone and lower mantle (Lynner \& Long 2014, 2015). Tomographic studies that include higher mode surface wave dispersion and/or long period body waveforms generally result in models with better depth resolution, but lower lateral resolution. Several recent publications have reported seismic anisotropy of up to a few percents in the transition zone (e.g. Panning \& Romanowicz 2006a; Yuan \& Beghein 2013; Auer et al. 2014; Moulik \& Ekström 2014; Yuan \& Beghein 2014; Chang et al. 2015; Ferreira et al. 2019), but there are large discrepancies between models at those depths (Schaeffer et al. 2016). Differences in the models can arise from differences in the datasets, but also from different inversion techniques and applied regularizations.

Tomographic inverse problems are typically solved by trying to minimize a cost function that measures the distance between data and model predictions in a least-squares sense. They are, however, generally not well-posed and can thus be highly non-unique, i.e., several solutions can explain the data equivalently well. Traditional inverse methods deal with this non-uniqueness by imposing subjective regularizations, but those can have significant impacts on the calculated solution. These regularizations include the choice of the reference model, the model parameterization, and/or the choice of ad hoc damping parameters that compromise between minimizing the misfit and the size of the model (Trampert 1998). In addition, regularized inversions can underestimate model uncertainties as the posterior model covariance is generally smaller than the prior by construction (Tarantola 2005). In addition, resolution analyses for regularized inverse methods depend on the regularization, which may give a false sense of certainty in some model parameters. It should also be noted that errors in tomographic models can be introduced by errors or approximations in the forward theory. One common example in seismic tomography is solving linearized equations instead of the full non-linear problem.

In this study, in order to avoid some of these issues, we developed a Hierarchical Transdimensional Bayesian (TB) inverse method (Sambridge et al. 2005; Bodin et al. 2012, 2016; Gao \& Lekić 2018), which uses a reversible jump Monte Carlo Markov Chain (rj-MCMC) algorithm (Green 1995, 2003; Gallagher et al. 2009) to solve the non-linear forward problem associated with calculating higher mode surface wave dispersion from an interior model. With advances in computing, Monte Carlo methods, which repetitively solve the forward problem, have become more and more common in geophysics and seismic tomography in particular. An 
advantage of these techniques is that they can solve non-linear problems directly instead of relying on a linearized approximation of the problem. In addition, besides the choice of the prior model parameter distribution, they do not require as much regularization as traditional inverse methods. By testing thousands or millions of possible models, they can provide an ensemble of solutions that fit the data instead of choosing a single one with a subjective regularization. This provides quantitative posterior model uncertainties and allows for an estimate of the probability of different values of the model parameters. In our study, we used the resulting parameter distributions to determine the likelihood of the presence of radial anisotropy at different depths in the upper mantle.

It should be noted that a different fully non-linear approach to the same problem was previously taken by Visser et al. (2008a) to determine the likelihood of radial anisotropy in the top $1400 \mathrm{~km}$ of the mantle at the global scale. They had found that $1-2 \%$ radial anisotropy with vertically polarized shear-waves traveling faster than horizontally polarized shear-waves was likely in the mantle transition zone globally. Our technique has, however, several possible advantages compared to their work. First, it allows for the depth parametrization to change during the model space search, letting the data guide how complicated the model needs to be instead of imposing a fixed set of basis functions a priori. Second, the hierarchical nature of our MCMC algorithm enables us to include the data noise among the unknowns. Using incorrect data noise can negatively impact the accuracy of inversion results (Bodin et al. 2012). Instead, by inverting for data noise, we allow the algorithm to decide how much noise is needed in order to explain the measurements without overfitting them.

In this manuscript, we first present the data and method employed. Second, we demonstrate the dependence of radial anisotropy models on the regularization imposed when performing linearized inversions. Third, we apply our TB technique to a fundamental and higher mode surface wave dataset sampling the Indian Ocean and compare the results with those of a regularized inversion. Fourth, we test the performance of the method with synthetic tests. Finally, we briefly discuss the results before drawing conclusions.

\section{DATA}

The measurements used in this study are the Rayleigh and Love wave fundamental and higher mode phase velocity maps measured by Visser et al. (2008b). The authors used a model space search to perform those measurements, which allowed them to also calculate quantitative data uncertainties. Both Rayleigh and Love waves are necessary to constrain radial anisotropy as they each are sensitive to separate shear-wave velocity polarization directions. Rayleigh waves 
are sensitive to vertically polarized shear-wave speed $\left(V_{S V}\right)$, and Love waves are sensitive to horizontally polarized shear-wave speed $\left(V_{S H}\right)$. The Voigt average bulk shear-wave speed $\left(V_{S}\right)$ and radial anisotropy $(\xi)$ can be calculated from $V_{S V}$ and $V_{S H}$ (Babuska \& Cara 1991):

$$
\begin{aligned}
V_{S} & =\sqrt{\left(2 V_{S V}^{2}+V_{S H}^{2}\right) / 3} \\
\xi & =V_{S H}^{2} / V_{S V}^{2}
\end{aligned}
$$

Both wave types are also sensitive to density, $\rho$, and Rayleigh waves are additionally sensitive to elastic parameters relating to compressional wave velocities. However, due to the existence of parameter trade-offs, Rayleigh waves and Love waves can only resolve shear-wave parameters. We thus only inverted for the two S-wave velocity parameters only, $V_{S H}$ and $V_{S V}$, as explained in the Method section.

Visser et al. (2008b) measured phase velocity dispersion for branches up to the sixth overtone for Rayleigh waves and up to the fifth overtone for Love waves. Including higher mode data is critical to constrain elastic structure beyond the uppermost $200 \mathrm{~km}$ of the mantle because they are sensitive to deeper structure than fundamental modes at the same period. Sensitivity curves for $V_{S}$ and $\xi$ for this dataset are displayed in Fig. 1 at periods between $35 \mathrm{~s}$ and $175 \mathrm{~s}$. They show that the higher modes are much more sensitive to structure below $200 \mathrm{~km}$ than the fundamental modes, and demonstrate that the dataset used in this study is sensitive to structure well below the bottom of the mantle transition zone. For this work, we divided the phase velocity maps into $5^{\circ} \times 5^{\circ}$ cells, with each cell being assigned velocities based on the values of the maps at the center of the grid cell. The surface wave velocities were inverted at each of the resulting 238 grid cells separately using different methods, as described in section 3, to obtain one-dimensional (1-D) shear-wave velocity depth profiles. The obtained individual 1-D velocity profiles were then used to construct shear-wave velocity and anisotropy maps.

\section{METHOD}

In this study, we compared results from regularized linear inversions of phase velocity maps with those obtained with a fully non-linear Bayesian approach. Both methods are described below. 

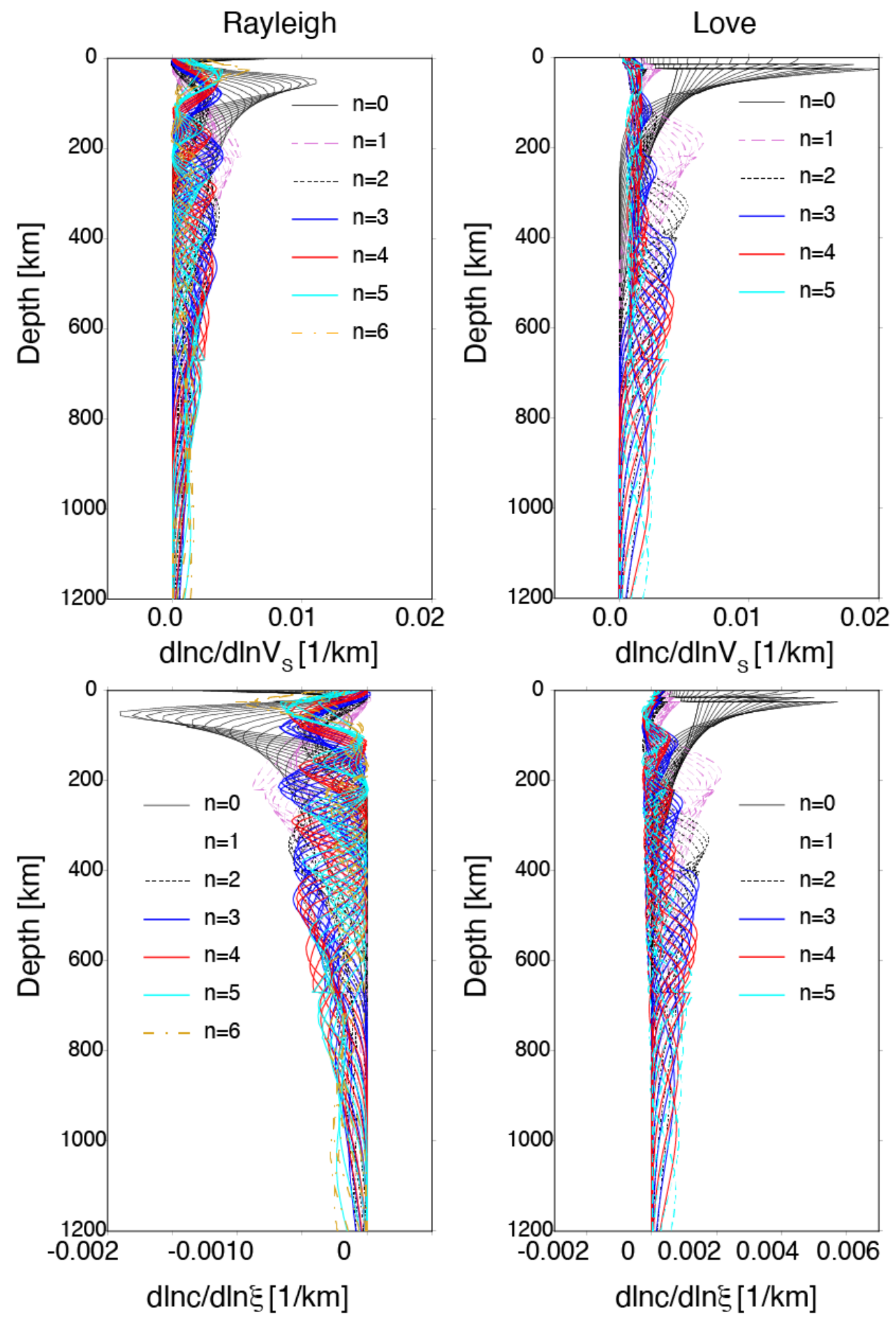

Figure 1. Rayleigh (left) and Love (right) wave phase velocity $(c)$ sensitivity kernels for perturbations in $V_{S}$ (top) and $\xi$ (bottom) between $35 \mathrm{~s}$ and $175 \mathrm{~s}$ period for fundamental $(n=0)$ and higher modes $(n>0)$. 


\subsection{Regularized Inversion}

The relation between perturbations in phase velocity, $c$ and perturbations in the parameters m describing the physical state of Earth's interior can be written as follows (Woodhouse \& Dahlen 1978):

$$
\delta d(T)=\int_{0}^{R} K_{\mathbf{m}}(T, r) \delta \mathbf{m}(r) d r+\sum_{b} \delta d_{b} K_{b}(T)
$$

where $R$ is the radius of the Earth, $\delta d$ represents the phase or group velocity data, $T$ is the period considered, and $b$ is the boundary radius. $K_{\mathbf{m}}(T, r)$ is a sensitivity kernel that maps perturbations to the elastic model $\mathbf{m}$ at depth $r$ to velocity change. $K_{b}$ is a boundary kernel for internal boundary $b$ that relates perturbations in the depth $d_{b}$ of the boundary to a perturbation in phase velocity. The vector $\mathbf{m}$ contains all the model properties, i.e. density and elastic parameters, and a sum over each one of them is implicit. $K_{\mathbf{m}}$ are sensitivity kernels, or partial derivatives, for model perturbations $\delta \mathbf{m}$. An identical relation exists for group velocities.

When constraining radial anisotropy, one needs to account for five elastic parameters in addition to density. These elastic parameters are : $L$ and $N$, which relate to the speed of vertically and horizontally polarized shear-waves, respectively $\left(V_{S V}^{2}=L / \rho\right.$ and $\left.V_{S H}^{2}=N / \rho\right)$; $C$ and $A$, which relate to $\mathrm{P}$-wavespeed for waves traveling vertically and horizontally, respectively $\left(V_{P V}^{2}=C / \rho\right.$ and $\left.V_{P H}^{2}=A / \rho\right)$; and $F$, which describes waves traveling at intermediate angle. If we define $\eta=F /(A-2 L)$, we thus have (Montagner 1986):

$$
\begin{aligned}
& \delta d(T)=\int_{0}^{R}\left[\quad K_{V_{S V}}(T, r) \delta V_{S V}(r)+K_{V_{S H}}(T, r) \delta V_{S H}(r)\right. \\
& +K_{V_{P V}}(T, r) \delta V_{P V}(r)+K_{V_{P H}}(T, r) \delta V_{P H}(r) \\
& \left.+K_{\eta}(T, r) \delta \eta(r)+K_{\rho}(T, r) \delta \rho(r)\right] d r+\sum_{b} \delta d_{b} K_{b}(T)
\end{aligned}
$$

Among these six parameters, only the two shear-wave related parameters can be reasonably well resolved with surface waves (e.g. Beghein \& Trampert 2004). It is customary to assume scaling relationships for the other parameters and to invert for the shear-wave parameters only. Such relationships were derived by Montagner \& Anderson (1989) for the uppermost $200 \mathrm{~km}$ of the mantle and are commonly used in surface wave inversions for radial anisotropy (e.g. Panning et al. 2006; Xing \& Beghein 2015):

$$
\begin{aligned}
\frac{\delta \ln V_{P}}{\delta \ln V_{S}} & =0.5 \\
\frac{\delta \ln \rho}{\delta \ln V_{S}} & =0.33 \\
\frac{\delta \ln \phi}{\delta \ln \xi} & =-2.5
\end{aligned}
$$




$$
\frac{\delta \ln \eta}{\delta \ln \xi}=-1.5
$$

While these relations are strictly only valid for the shallow upper mantle, studies have shown that using them for the deeper mantle does not affect the shear-wave velocity or anisotropy model significantly (Panning et al. 2006). We thus applied them to the entire depth range of interest. Equation 3 then becomes:

$$
\delta d(T)=\int_{0}^{R}\left[K_{V_{S V}}^{\prime}(T, r) \delta V_{S V}(r)+K_{V_{S H}}^{\prime}(T, r) \delta V_{S H}(r)\right] d r+\sum_{b} \delta d_{b} K_{b}(T)
$$

where the $K^{\prime}$ kernels are linear combinations of the kernels in Equation 4. The problem can alternatively be described in terms of perturbations in bulk shear-wave velocity and anisotropy:

$$
\delta d(T)=\int_{0}^{R}\left[K_{V_{S}}(T, r) \delta V_{S}(r)+K_{\xi}(T, r) \delta \xi(r)\right] d r+\sum_{b} \delta d_{b} K_{b}(T)
$$

where $V_{S}$ and $\xi$ are defined in Equations 1 and 2.

These equations can be discretized by introducing a basis function to parameterize the depth dependence of the model parameters. We chose a basis function in terms of $J$ splines $S_{j}:$

$$
\mathbf{m}(r)=\sum_{j=1}^{J} \mathbf{m}^{j} S_{j}(r)
$$

Equation 3 then becomes:

$$
\delta d(T)=\sum_{i=1}^{2} \sum_{j=1}^{J} m_{i}^{j} \int_{0}^{R} K_{m_{i}}(T, r) S_{j}(r) d r+\sum_{b} \delta d_{b} K_{b}(T)
$$

where index $i$ is for each one of the two elastic parameters we solve for. The forward problem can thus be written as:

$$
\mathbf{d}=\mathbf{G m}
$$

where $\mathbf{d}$ is the data vector. $\mathbf{G}$ is the kernel matrix that maps the model space into the data space:

$$
G_{k j}=\sum_{i} \int_{0}^{R} K_{m_{i}}^{k}(r) S_{j}(r) d r
$$

with $G_{k j}$ an element of $\mathbf{G}$, and $K_{m_{i}}^{k}(r)$ the sensitivity kernel for the ith model parameter in $\mathbf{m}$ and the kth measurement in $\mathbf{d}$.

A generalized inverse matrix, $\mathbf{G}^{g}$, can be calculated via singular value decomposition (SVD) and can be used to determine a least-squares solution m (Jackson 1972; Lanczos 1961; Wiggins 1972). If $\mathbf{G}$ is a $n \times m$ matrix where $n$ is the number of data points and $m$ is the number of model parameters, it can be decomposed into: 


$$
\mathbf{G}=\mathbf{U} \Lambda \mathbf{V}^{\mathbf{T}}
$$

where $\mathbf{U}$ is a $n \times n$ matrix of eigenvectors that span the data space, $\Lambda$ is a $n \times m$ diagonal matrix whose columns are non-negative eigenvalues $\lambda_{i}$, and $\mathbf{V}$ is a $m \times m$ matrix of eigenvectors that span the model space. The $\lambda_{i}^{2}$ are the singular values of $\mathbf{G}$. The generalized inverse of $\mathbf{G}$ is thus:

$$
\mathbf{G}^{g}=\mathbf{V}_{p} \boldsymbol{\Lambda}_{p}^{-1} \mathbf{U}_{p}^{T}
$$

where $p$ is the number of non-zero eigenvalues. The estimated model parameters $\mathbf{m}^{e s t}$ are then given by a sum limited to the non-zero eigenvalues to insure stability of the solution:

$$
\mathbf{m}^{e s t}=\mathbf{G}^{g} \mathbf{d}
$$

Here, we applied the method of Matsu'Ura \& Hirata (1982) to determine $p$ : we first normalize $\mathbf{G}$ by the data covariance matrix $\mathbf{C}_{d}$ and a prior model covariance matrix $\mathbf{C}_{m}$ :

$$
\mathbf{G}^{\dagger}=\mathbf{C}_{d}^{-1} \mathbf{G} \mathbf{C}_{m}
$$

We then calculated its eigenvalues and determined the solution using all the eigenvalues smaller or equal to unity. For $\mathbf{C}_{d}$, we used a diagonal matrix and the uncertainties estimated by Visser et al. (2008b). The choice of model covariance matrix acts as implicit regularization as it yields a different cutoff in the number of eigenvalues (Snieder \& Trampert 2000).

In this study, we inverted Love and Rayleigh waves jointly using the SVD technique described above, and compared models obtained with different prior values for $\mathbf{C}_{m}$ and with different model parameterizations (Equations 9 and 10). The misfit $\left(\phi_{d}\right)$ was defined by:

$$
\phi_{d}=\frac{1}{N} \sum_{i=1}^{N}\left(d_{i}^{o b s}-d_{i}^{p r e}\right)^{2},
$$

where $N$ is the total number of measurements, $d_{i}^{\text {obs }}$ is the $i$ th measured data, and $d_{i}^{\text {pre }}$ is the $i$ th predicted data calculated with Equation 12. We followed Xing \& Beghein (2015) and corrected for the effect of the crust by applying non-linear crustal corrections based on the 3-D crustal model CRUST 1.0 (Laske et al. 2013). To calculate these non-linear crustal corrections, we divided the phase velocity maps into $5^{\circ} \times 5^{\circ}$ cells and, at each grid cell, we created local 1-D models composed of CRUST1.0 and the PREM mantle. At each grid cell, we then used MINEOS (Masters et al. 2011) to predict phase velocities. Those predictions were then subtracted from the real measurements so that the corrected data contain information about mantle structure only. Sensitivity kernels were calculated at each grid cell relative to the local reference model, and we inverted for perturbations in mantle structure only. 


\subsection{Transdimensional Hierarchical Bayesian method}

While solving the inverse problem using the regularized method described above is relatively simple and efficient, it yields solutions that depend on the regularizations imposed, including the choice of a reference model. In addition, posterior model uncertainties can be underestimated if the model null-space is large (Trampert 1998), and the non-linear relation between phase velocity and structure are approximated by linearized equations. We thus developed instead a non-linear model space search approach to find a range of plausible solutions instead of choosing one with a subjective regularization. Our technique is based on the hierarchical TB method of Bodin et al. (2012) to explore the model space with a rj-MCMC method, which we combined with Fortran code MINEOS (Masters et al. 2011) to solve the forward problem. As stated before, some of the advantages of this method compared to traditional inversions include the fact that the depth parameterization can change at each iteration, allowing the data themselves to constrain how complicated the model needs to be. In addition, unknown data noise can be simultaneously inverted for instead of being fixed at a presumed level, which reduces the risk of mapping noise into the model. Another advantage is that model parameters are described by probability density functions (PDFs), which can be used to quantify their uncertainties.

In this study, noise was assumed to be Gaussian, and we chose to parameterize the models as a set of layers. For each layer, a Voronoi nuclei defines its elastic parameter, and the boundaries between layers are the midpoints between adjacent nuclei. The number of points and their depths are allowed to change at each iteration, meaning that the model depth parameterization is a variable of the inversion, in addition to the elastic parameters for each layer. Monte Carlo inversions are generally time-consuming, and this is especially true when solving the forward problem with MINEOS. Minimizing the number of unknowns to solve for is thus important. For this purpose, we inverted Love and Rayleigh waves separately for $V_{S H}$ and $V_{S V}$, respectively. We verified that the models obtained were equivalent to joint inversion results (see section 5.1). Scaling relations were imposed on the other elastic parameters as in section 3.1 (Montagner \& Anderson 1989). The resulting distributions of 1-D $V_{S H}$ and $V_{S V}$ profiles were then sampled and used to calculate $V_{S}$ and $\xi$ distributions (Equations 1 and 2).

With a hierarchical TB approach, model parameters or data noise level are perturbed iteratively, and each new model and data noise combination is either accepted or rejected based on an acceptance criteria as described below. If a change is accepted, the iteration repeats with the modified parameter, and a parameter is randomly selected to be perturbed again. If rejected, the iteration repeats with the model and noise used prior to the modification. 
Every 100 iterations, the current model is added to the ensemble of solutions. This process is repeated thousands of times in order for the chain to reach convergence, after which the saved ensemble of models gives a posterior for $V_{S H}$ or $V_{S V}$. For our application, each chain had a burn-in period of 50,000 iterations before models were saved. We then ran enough chains and iterations to get 10,000 samples after the burn-in period.

Data noise perturbations consisted in random values generated from a prior Gaussian distribution that were added to the original noise following level. A model perturbation can consist in moving a Voronoi nucleus, inserting or removing a nucleus, or randomly modifying the elastic parameters at a nucleus using a prior Gaussian distribution. The acceptance or rejection criteria used depends on the likelihood and the prior probability as described in Bodin et al. (2012). According to Bayes' theorem (Bayes 1763), the posterior probability of having model $\mathbf{m}$ when a set of observed data $\mathbf{d}_{o b s}$ is fixed was given by:

$$
p\left(\mathbf{m} \mid \mathbf{d}_{o b s}\right) \propto p\left(\mathbf{d}_{o b s} \mid \mathbf{m}\right) p(\mathbf{m}),
$$

where $p\left(\mathbf{d}_{\text {obs }} \mid \mathbf{m}\right)$ is the likelihood function, which gives the probability of observing data given a particular model, and $p(\mathbf{m})$ is the a priori probability of model $\mathbf{m}$, which reflects our prior knowledge on the parameters before having the observed data. The acceptance criteria compares the posterior for the perturbed model to that of the original model. The larger the perturbed model posterior, the higher the likelihood the new model will be accepted. Here, the likelihood function is defined by:

$$
p\left(\mathbf{d}_{o b s} \mid \mathbf{m}\right)=\frac{1}{\sqrt{\left(2 \pi \sigma^{2}\right)^{N}}} e^{\frac{-\left(\mathbf{d}_{p r e}-\mathbf{d}_{o b s}\right)^{2}}{2 \sigma^{2}}}
$$

where $\mathbf{d}_{\text {pre }}$ are the data predicted by model $\mathbf{m}, N$ is the total number of measurements, and $\sigma$ is the total data noise level. The prior can be written as:

$$
p(\mathbf{m})=p(\mathbf{c}, \mathbf{v} \mid k) p(k) p(\mathbf{h})
$$

where $\mathbf{c}$ is the set of depths of the Voronoi nuclei associated with $\mathbf{m}, \mathbf{v}$ is the set of wave velocities, $k$ is the number of Voronoi nuclei, and $\mathbf{h}$ is the set of noise parameters.

In Bodin et al. (2012), the prior $p(\mathbf{c}, \mathbf{v})$ was a uniform distribution that was constant with depth. Thus, $p(\mathbf{c}, \mathbf{v} \mid k)$ can be written as the product of $p(\mathbf{c} \mid k)$ and $p(\mathbf{v} \mid k)$. The prior probabilities of models with the same number of Voronoi nuclei are equal, but they differ when layers are added and removed due to the prior on the velocities:

$$
p(\mathbf{v} \mid k) \quad=\prod_{i=1}^{k} p\left(v_{i} \mid k\right),
$$




$$
p\left(v_{i} \mid k\right)=\left\{\begin{array}{lr}
\frac{1}{\Delta v} & V_{\min } \leq v_{i} \leq V_{\max } \\
0 & \text { otherwise }
\end{array}\right.
$$

where $v_{i}$ is the velocity of the $i$ th Voronoi nuclei and $\Delta v$ is the range of allowable velocities. In our case, the prior on $V_{S V}$ or $V_{S H}$ is a uniform distribution within $10 \%$ of CRUST1.0 in the crust and within $10 \%$ of PREM in the mantle. Since velocities are generally increasing with depth, $\delta v$ increases with depth. This depth-dependent prior means that points at shallower depths will generally have a higher prior probability than points at larger depths.

\section{LINEAR INVERSIONS RESULTS}

\subsection{Effect of Regularization}

Figs. 2 and 3 illustrate how the choice of parameterization and regularization can affect the models in the case of a traditional inversion method using linearized equations. In one case, we parameterized the models in term of perturbations in $V_{S}$ and $\xi$ relative to PREM (Equation 10), and in the other case we tested a parameterization in terms of $V_{S V}$ and $V_{S H}$ perturbations (Equation 9), and calculated $d \ln V_{S}$ and $\xi$ using Equations 1 and 2. We changed the regularization by modifying the prior model covariance matrix $\mathbf{C}_{m}$ (Equation 18), which resulted in different numbers of independent parameters. Increasing the prior model variance is equivalent to decreasing a damping factor (Snieder \& Trampert 2000) and increases the number of independent parameters.

For $V_{S}$ at $100 \mathrm{~km}$ depth (Fig. 2), increasing the number of independent parameters does not significantly affect the strengths of the anomalies or their overall distribution across the study region. In both cases shown, oceanic ridges are associated with negative velocity anomalies and Africa, and Madagascar are characterized by positive velocity anomalies. We found, however, that changing the parameterization affects the strength of the relatively low velocities located under and near mid-ocean ridges. For a fixed number of independent parameters, the parameterization in terms of $\xi$ and $V_{S}$ shows stronger negative $d \ln V_{S}$ than the parameterization in terms of $V_{S V}$ and $V_{S H}$ at $100 \mathrm{~km}$ depth. In addition, the change in parameterization produces different results under Madagascar, Somalia, and the southwestern part of the Indonesian subduction zone: Inversions for $V_{S H}$ and $V_{S V}$ yield positive $d \ln V_{S}$ under these regions, whereas inversions for $V_{S}$ and $\xi$ yield negative $d \ln V_{S}$.

For anisotropy, the models are even more sensitive to changes in parameterization and regularization, even at $100 \mathrm{~km}$ depth where the data have some of the strongest sensitivity to structure (Fig. 1). Fig. 3 shows that for the $V_{S V}$ and $V_{S H}$ inversions at $100 \mathrm{~km}$ depth, both 

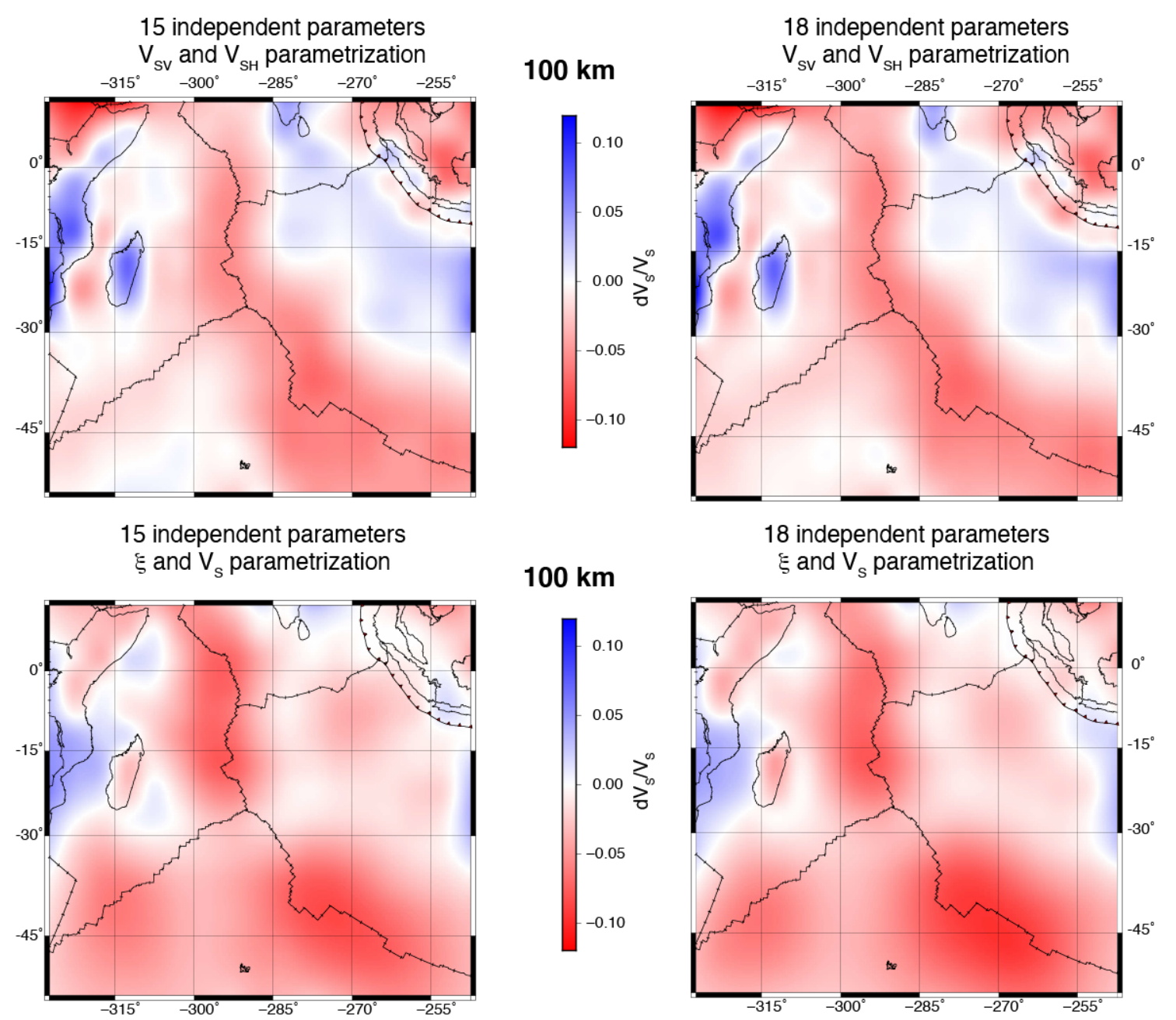

Figure 2. $d \ln V_{S}$ model at $100 \mathrm{~km}$ depth resulting from linearized inversions with 15 (left) and 18 (right) independent parameters. Top: $d \ln V_{S}$ calculated from inversions parameterized in terms of $V_{S V}$ and $V_{S H}$ perturbations. Bottom: $d \ln V_{S}$ resulting from inversions parameterized in terms of $d \ln V_{S}$ and $d \ln \xi$.

regularizations give $\xi<1$ (i.e. $V_{S V}>V_{S H}$ ) at several locations, including under Madagascar, eastern Africa, and along the Central Indian Ridge. On the contrary, with the $\xi$ and $V_{S}$ parameterization, most of the study area is characterized by $\xi<1$ (i.e. $V_{S V}<V_{S H}$ ). This dependence of the anisotropy model on regularization and parameterization is an indication that the model null-space and thus the model uncertainties are likely large. Our tests also demonstrate how the prior choice of parameterization can affect the results. 

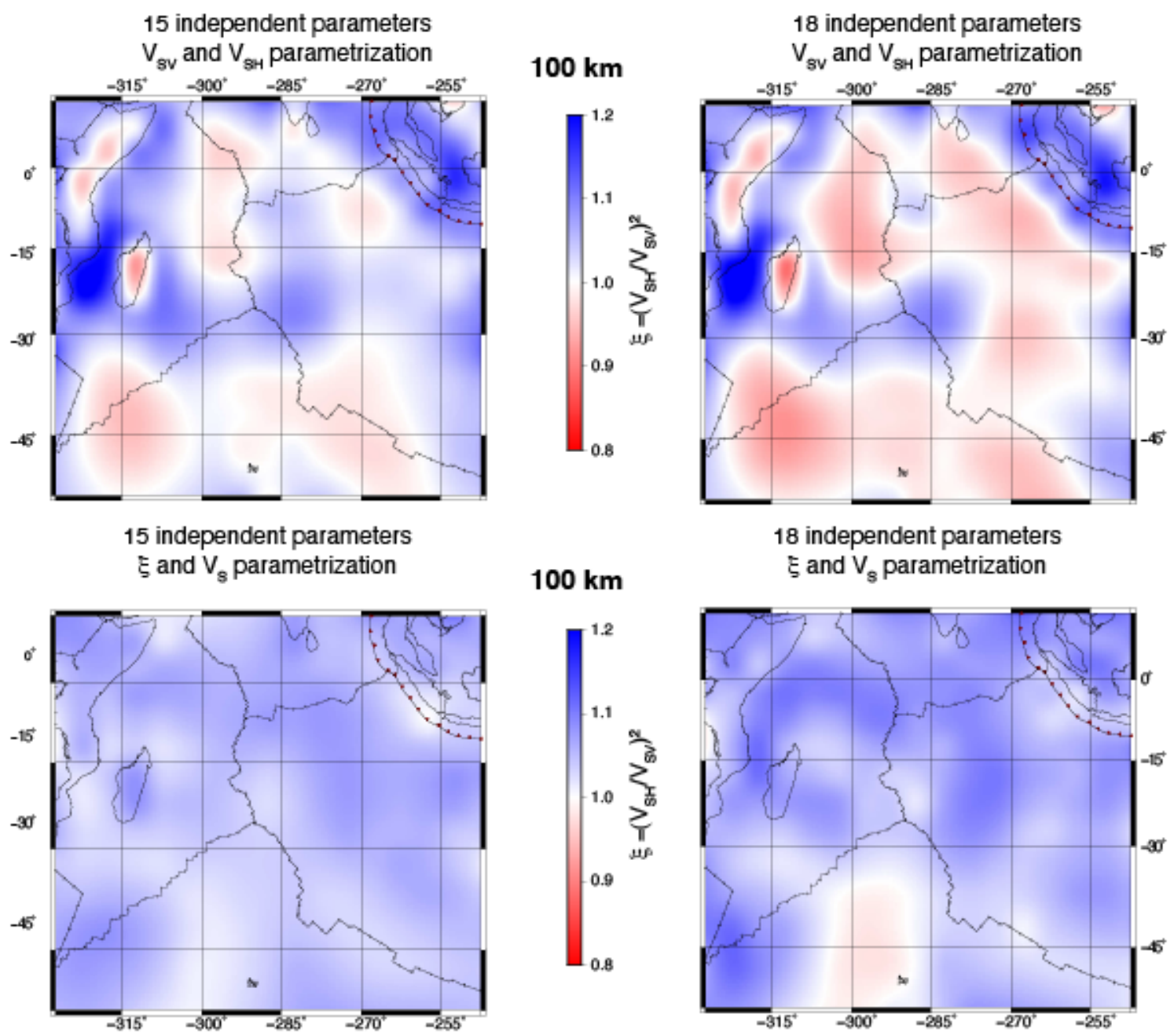

Figure 3. $\xi$ model at $100 \mathrm{~km}$ depth resulting from linearized inversions with 15 (left) and 18 (right) independent parameters. Top: $\xi$ calculated from inversions parameterized in terms of $V_{S V}$ and $V_{S H}$ perturbations. Bottom: $\xi$ resulting from inversions parameterized in terms of $d \ln V_{S}$ and $d \ln \xi$.

\subsection{Effect of Mantle Transition Zone Topography}

In theory, topography of the MTZ boundaries can affect higher mode phase velocities (Equation 12). Higher modes have thus the potential to contain information about the MTZ thickness, which could be used to constrain its thermochemical state (Meier et al. 2009). Here, we tested the effect of MTZ topography on our results by first inverting the dispersion data provided by Visser et al. (2008b) and corrected for the effect of the crust as described above, and then comparing the models with those obtained by inverting the same data set corrected with a prior model of MTZ topography. For this, we used the $410 \mathrm{~km}$ and $670 \mathrm{~km}$ topography values obtained by Huang et al. (2019) with SS precursors and calculated their contributions 
to Rayleigh and Love wave phase velocities (Equation 12). Fig. S1 displays examples of MTZ topography corrections for MTZ sensitive modes and shows that they are at least an order of magnitude smaller than the measurements in the study region, and are thus unlikely to have an effect on the models. These contributions were nevertheless removed from the dispersion measurements and the remaining signal was inverted for at several grid cells.

Fig. S2 shows the 1-D $V_{S}$ and $\xi$ profiles obtained at two different locations within the study area using data corrected for the crust and data corrected both for the crust and for deviations in the depth of the $440 \mathrm{~km}$ and $660 \mathrm{~km}$ boundaries. We see that the effect of the boundary topography on the model velocities is not significant as both sets of inversions produced models that were nearly identical. This is in agreement with the study of Meier et al. (2009) who inverted these same higher mode dispersion measurements using neural networks and found that the $410-\mathrm{km}$ and $660-\mathrm{km}$ topographies were not well constrained with respect to the prior PPDFs. We thus proceeded with uncorrected data for the rest of the study.

\section{NON-LINEAR INVERSION RESULTS}

\subsection{Joint vs. Separate Inversions}

Non-linear inversions with Monte Carlo methods are computationally prohibitive. This is especially true for joint Love and Rayleigh wave inversions as they double the number of unknowns compared to separate inversions. Previous studies have shown that Love and Rayleigh wave velocities are sufficiently independent of each other to justify separate inversions since Love waves are mostly sensitive to $V_{S H}$ and Rayleigh waves are mostly sensitive to $V_{S V}$. However, most of those studies were based on regularized linear inversions (e.g. Ekström \& Dziewonski 1998) and may not apply to our study. An exception is the work of Visser et al. (2008b) who used a fully non-linear Monte Carlo approach to invert for radial anisotropy at the global scale. By separating the anisotropic PREM model into a horizontally polarized model and a vertically polarized model, the authors verified that dispersion curves calculated for Love and Rayleigh waves separately assuming isotropy did not differ significantly from those calculated for anisotropic PREM. The differences were within data errors. They did not, however, compare joint and separate non-linear inversions of real dispersion data. For completeness, we thus decided to verify whether this assumption is valid for our method using real data.

For this purpose, we inverted Love and Rayleigh wave fundamental and higher mode dispersion data separately at a few grid cells, and compared the models with those obtained from joint Love and Rayleigh wave TB inversions (Fig. 4). An important aspect of these joint 
inversions is that they were performed using independent depth parameterizations for $V_{S H}$ and for $V_{S V}$. As demonstrated by Gao \& Lekić (2018), inversions performed in a TB framework that use identical choices of depth parameterizations for the different unknowns can bias structure estimates. This "attached-type" parameterization is very common in seismology and has the advantage of potentially reducing the number of parameters, but it also constitutes a form of implicit regularization that imposes the same geometry to all parameters. It often results in a model geometry that is determined by the best resolved parameters but that does not necessarily appropriate for the others. We verified that this is true for our dataset as well. Fig. 4 compares results of joint inversions with independent parametrizations for $V_{S H}$ and for $V_{S V}$ and results of separate Love and Rayleigh wave inversions using our non-linear TB inversion approach. It displays the mean models and their $90 \%$ confidence intervals on the same plots for the two types of inversions. We can see that there are few differences between the mean models and that separate inversions yield equivalent results to joint inversions as long as model uncertainties are accounted for. We thus proceeded with separate inversions for the rest of our study.

\subsection{Effect of noise parameterization}

As explained above, one advantage of the TB method is that it can invert for unknown data noise jointly with other parameters to prevent the mapping of data uncertainties into the model. While the phase velocity maps we used here (Visser et al. 2008b) were published with quantitative uncertainties at each period, these uncertainties resulted from averaging the errors on each measured earthquake-station pair. They therefore do not reflect the lateral variations in the uncertainties that can be caused by differences in ray coverage, for instance. It is thus reasonable for us to invert for data noise in addition to $V_{S}$ and $\xi$ instead of taking the values estimated by Visser et al. (2008b) at face value. However, assumptions have to be made about the prior noise distribution. On one hand, assuming that all the measurements have the same Gaussian noise adds only one unknown to the inverse problem and does not significantly increase the computational cost. This assumption may not, however, be valid, in which case any unmapped data noise can get mapped into the posterior distribution. On the other hand, assuming different noise levels for the different types of data can increase the computational cost of an already time-consuming technique and could increase trade-offs between resolving data noise parameters and model parameters.

In order to determine the best approach for our problem, we compared TB inversion results for different noise parameterizations. In the first case we used one noise parameter for the Love 

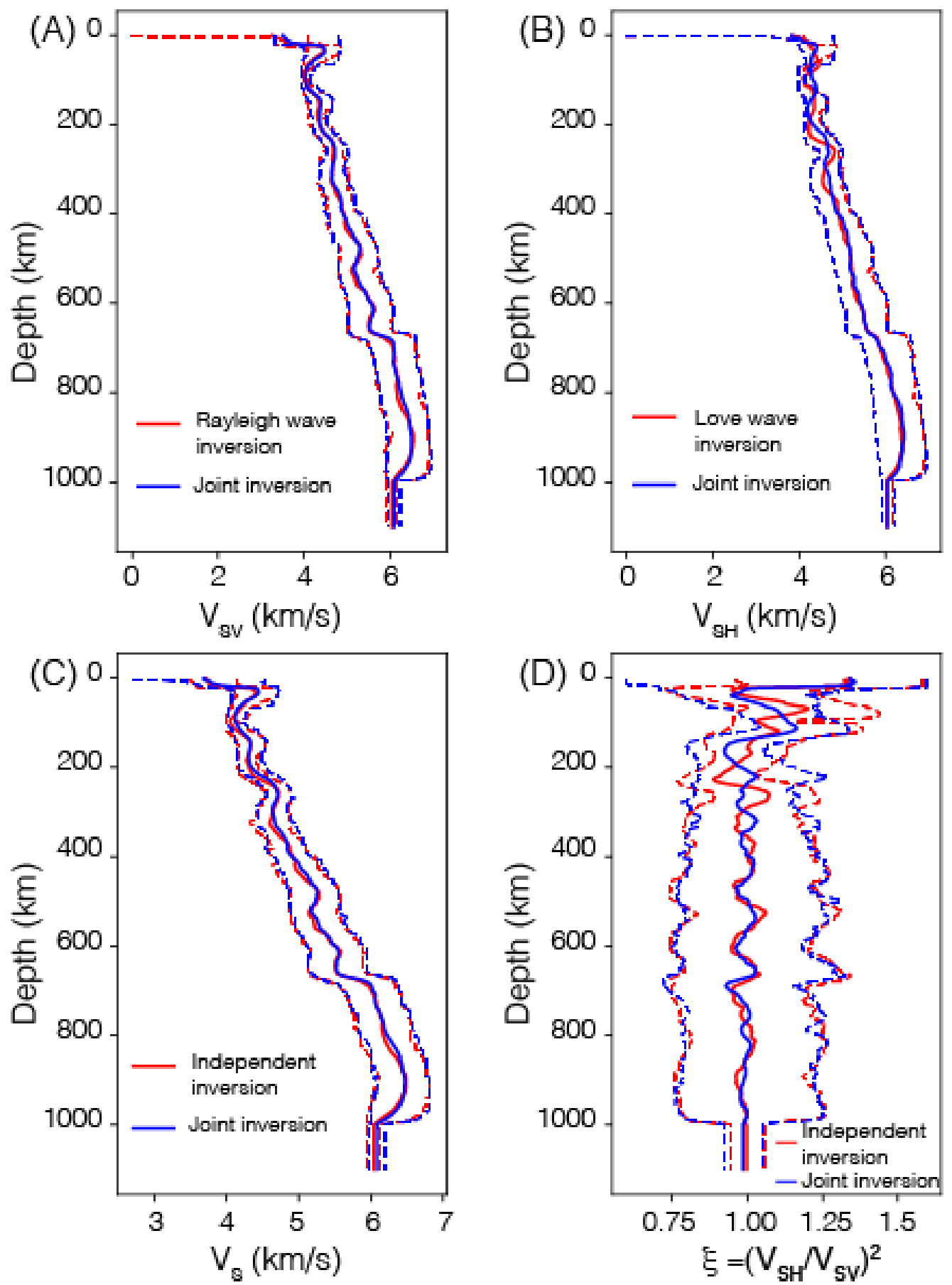

Figure 4. Comparison of $V_{S V}(\mathrm{~A})$ and $V_{S H}(\mathrm{~B})$ models obtained by independent and joint Love and Rayleigh wave inversions using our TB method at $\left(38^{\circ} \mathrm{S}, 79^{\circ} \mathrm{E}\right)$. The solid lines represent the mean model and the dashed lines represent their $90 \%$ confidence interval. (C) and (D) represent the resulting $V_{S}$ and $\xi$ models, respectively, and their $90 \%$ confidence interval. 
wave inversions and one for the Rayleigh wave inversions. In the second case, each of the data subsets (Love and Rayleigh) were divided into two groups: one group contains mode branches $n=0$ to $n=2$ and the other group contains the mode branches $n=3$ and higher. Each of these groups had its own noise level. In the third case, each mode branch within the two subsets had its own noise level. Results are shown for $V_{S V}$ and $V_{S H}$ in Fig. 5. Supplementary Figs. S3-S5 display results for the reconstructed $V_{S}$ and $\xi$ and the noise parameter PPDFs. The models are not significantly different, though the cases with additional noise parameters yielded less well-resolved $V_{S}$ and $\xi$. This is expected when increasing the number of unknowns in the inversion if trade-offs are present. However, the same general trends still exist across these different model distributions. We also note that the posterior noise parameters are generally larger for Love waves than for Rayleigh waves, indicating that Love wave dispersion data are noisier. Considering how little effect the number of noise parameters has on the velocity and anisotropy models, we proceeded using only one for each data set inversion.

\subsection{Models}

Results from both the linear and the TB inversions are shown in Figs. 6 and 7 for $V_{S}$ anomalies relative to PREM and for $\xi$, respectively. The TB results are the mean of the posterior model distributions at each grid cell. The data were not corrected for topography at the $410 \mathrm{~km}$ and $660 \mathrm{~km}$ boundaries since we showed that it does not have a significant effect on the models (Figs. S2). We see that at $100 \mathrm{~km}$ both methods result in negative $V_{S}$ perturbations under the Central Indian Ridge (CIR), the Southwestern Indian Ridge (SWIR), and Southeastern Indian Ridge (SEIR) branches. However, the two models disagree under Madagascar with the nonlinear inversion showing heterogeneous velocity perturbations and the linear inversion showing a positive anomaly. Similarly, some disagreement is found between the central Indian Ocean and the Indonesian subduction zone: the linear inversion results in a clear positive $d \ln V_{S}$ whereas the TB results display more heterogeneous anomalies. At larger depths, we see a strong reduction in the strength of the velocity anomalies, as often found in tomographic mantle models. At $200 \mathrm{~km}$, the SVD inversion shows a broad low velocity anomaly of 1$2 \%$ roughly following the oceanic ridges, with small regions of weak positive anomalies near southeastern Africa and around the Indonesian subduction zone. At that depth, the TB results also present evidence for $d \ln V_{S}<0$ along the CIR and SEIR of stronger amplitude (3-5\%) than in the SVD model, but the region is generally more heteregeneous than in the SVD model, including along the SWIR. At $400 \mathrm{~km}$, the TB method shows a ubiquitous $d \ln V_{S}>0$ while the SVD model is characterized by weak heterogeneities that include positive anomalies 

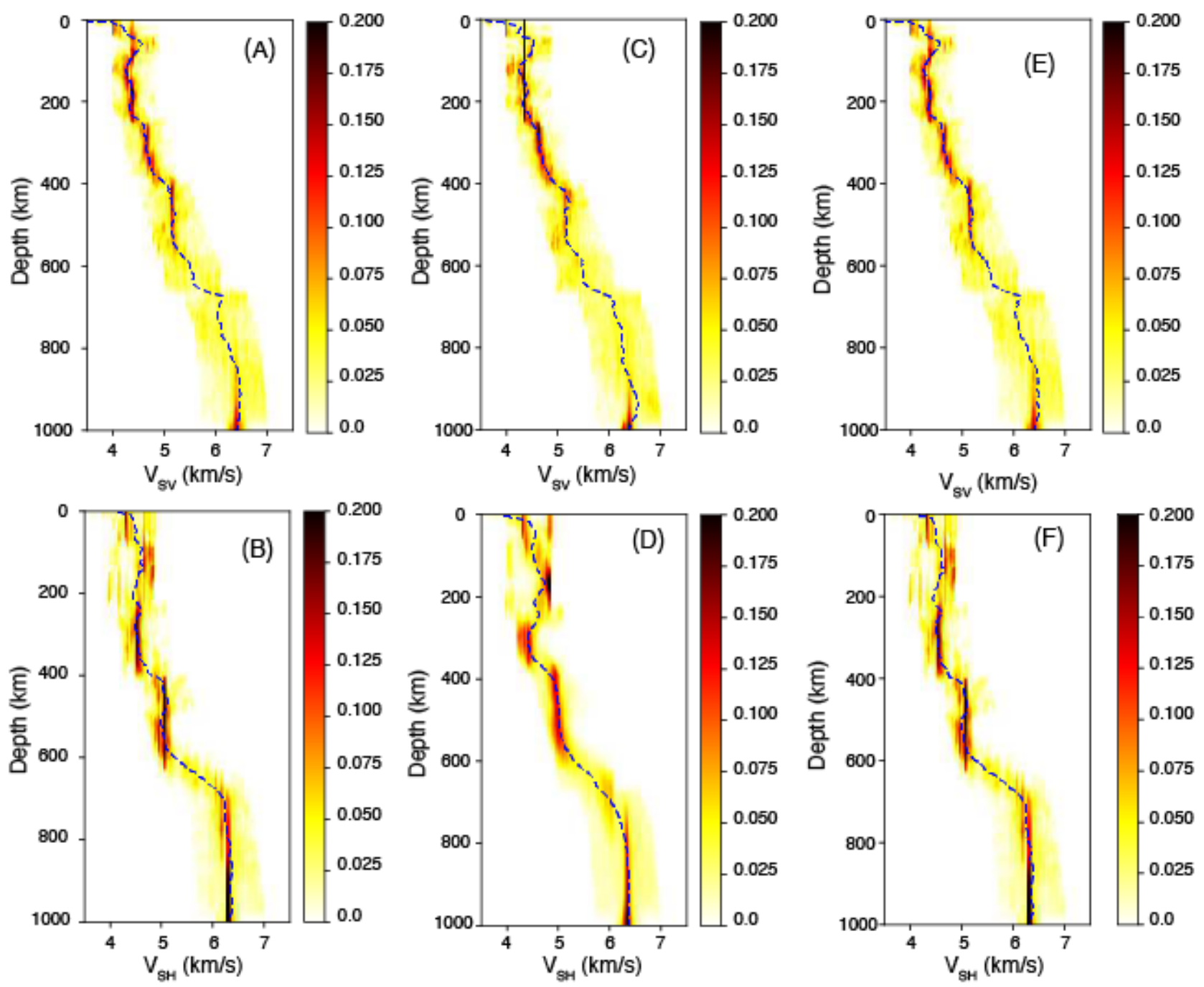

Figure 5. Comparison of inversion of Rayleigh (top) and Love (bottom) phase velocity data using a single noise parameter for all the modes ((A) and (B)), using one noise parameter for $n=0$ to $n=2$ and another one for $n \leq 3((\mathrm{C})$ and $(\mathrm{D}))$, and using one noise level per mode branch $((\mathrm{E})$ and $(\mathrm{F}))$. The dashed lines represent the mean models. The models shown here are for a grid cell at $\left(3^{\circ} \mathrm{S}, 79^{\circ} \mathrm{E}\right)$.

along the CIR and SEIR and along the Indonesian trench. In the upper MTZ, at $500 \mathrm{~km}$, both techniques show positive $d \ln V_{S}$ around Indonesia and west of the CIR, as well as negative $d \ln V_{S}$ east of the CIR and near the SWIR.

For radial anisotropy, the methods have less agreement (Fig. 7). At $100 \mathrm{~km} \mathrm{depth,} \mathrm{the}$ SVD inversion shows a general horizontally fast direction $(\xi>1)$ with pockets of vertically fast $(\xi<1)$ direction underneath the CIR, SWIR, and SEIR, and under Madagascar. The TB results display a more ubiquitous $\xi>1$ with larger amplitude across the region, with 

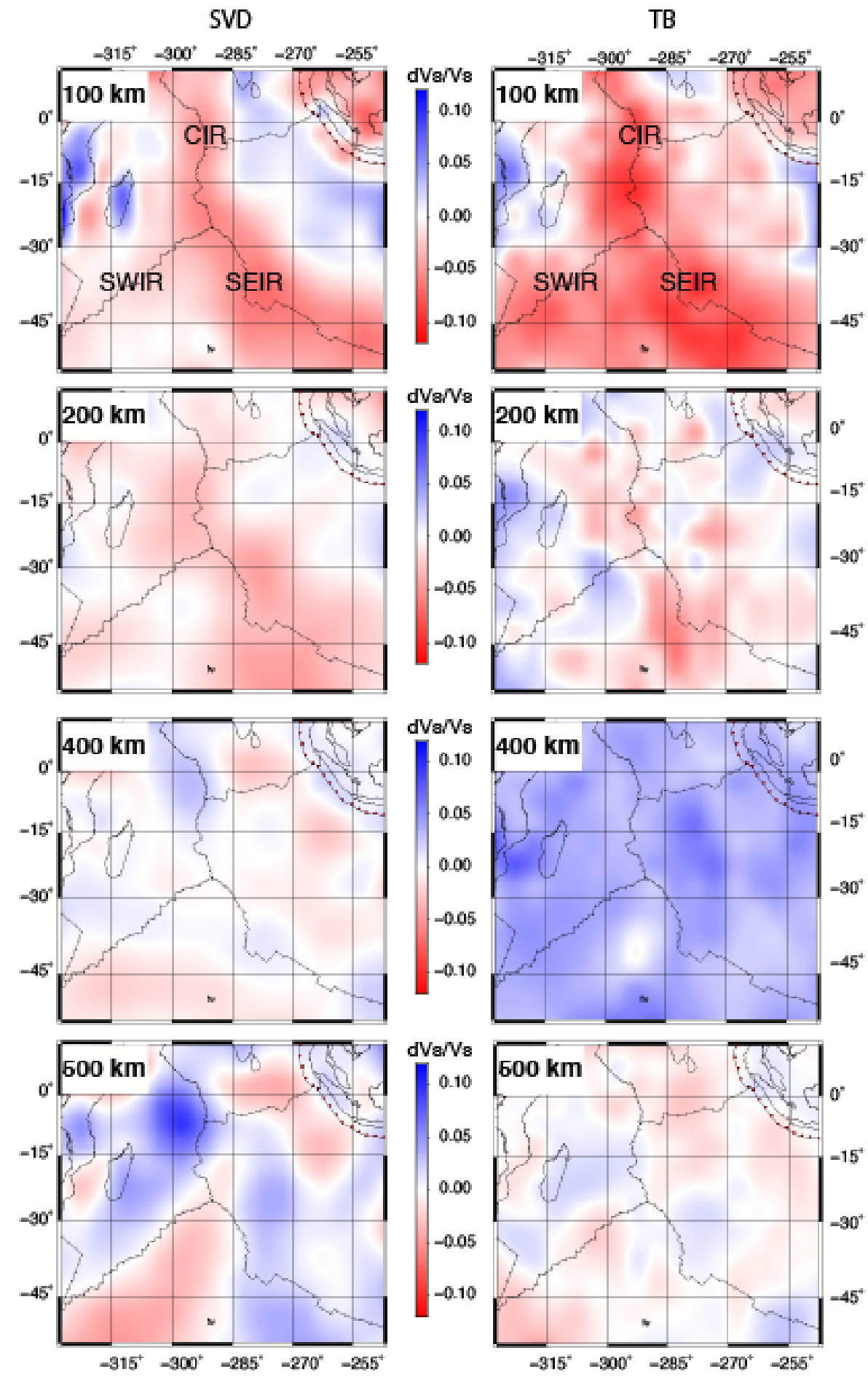

Figure 6. Bulk shear-wave velocity perturbations estimated from the SVD inversion (left) and from the mean of the posterior distribution obtained with the non-linear TB inversion (right). The Central Indian Ridge (CIR), Southwestern Indian Ridge (SWIR), and Southeastern Indian Ridge (SEIR) branches are labeled in the top panels for reference. 
the exception of a strongly vertically fast region south of the Indonesian subduction zone. At $200 \mathrm{~km}$, the linearized results show a horizontally fast zone throughout the region, peaking near the Rodrigues Triple Junction (RTJ). On the contrary, the TB results show a heterogeneous mixture of horizontally fast and vertically fast regions. At $400 \mathrm{~km}$, we found that, with the SVD inversion, most of the region is associated with $V_{S H}>V_{S V}$ except for a weak vertically fast anomaly along the CIR that was not visible at $200 \mathrm{~km}$. The TB results are different and more heterogeneous, and the strength of the $\xi<1$ anomalies is slightly greater than at $200 \mathrm{~km}$, whereas the $\xi>1$ anomalies appear to be weaker. This suggests that the TB inversion tends to favor a more vertically fast layer at that depth, as opposed to the SVD inversion, which yields a weaker anisotropy with horizontally fast shear-waves. In the MTZ, while details in the pattern of the anisotropy differ between the two techniques, both inversion methods favor mostly vertically fast shear-waves, including along the Indonesian subduction zone and the SWIR, and beneath Madagascar and Africa. Both models also suggest horizontally fast waves southwest of the Indonesian trench.

The TB method does not only offer a way to invert data with less assumptions than traditional methods, it also provides a way to quantify model parameter uncertainties. Representing uncertainties is nevertheless not simple as the Gaussian assumption one typically makes when plotting uncertainty maps may not be valid. While one can always try to fit a Gaussian through the posterior model distribution, it may give a false sense of uncertainty or certainty as it does not convey any skew, multiple peaks, or other non-Gaussian features. We thus decided instead to follow previous studies (Beghein \& Trampert 2006; Visser et al. 2008a) and use the posteriors to calculate the likelihood of the presence of positive or negative anomalies in the model parameters. These results are shown in Figs. 8 and 9 for $d \ln V_{S}$ relative to PREM and in Figs. 10 and 11 for radial anisotropy.

In Figs. 8 and 9 we see that at $100 \mathrm{~km}$ depth, the probability $P$ that $V_{S}$ anomalies are negative underneath oceanic ridges is greater or equal to 0.75 , and that the anomalies are of at least $1 \%$ in strength. In addition, while the negative anomalies beneath the SWIR are likely to be between $1 \%$ and $5 \%$, the CIR and SEIR are likely $(P \geq 0.75)$ characterized by stronger anomalies of at least $-5 \%$ at that depth. These relatively low velocity zones largely disappear at $200 \mathrm{~km}$ depth, with the exception of a likely $d \ln V_{S}<-1 \%$ underneath part of the SEIR. At this depth, positive relative velocity perturbations of at least $1 \%$ likely exist under East Africa and the SWIR, but they are unlikely to be $>5 \%$. At $400 \mathrm{~km}$, an almost ubiquitious $d \ln V_{s}>1 \%$ is found with a high likelihood. This anomaly is, however, probably lower than $5 \%$ for much of the region, with the exceptions of the region between Southern 
SVD

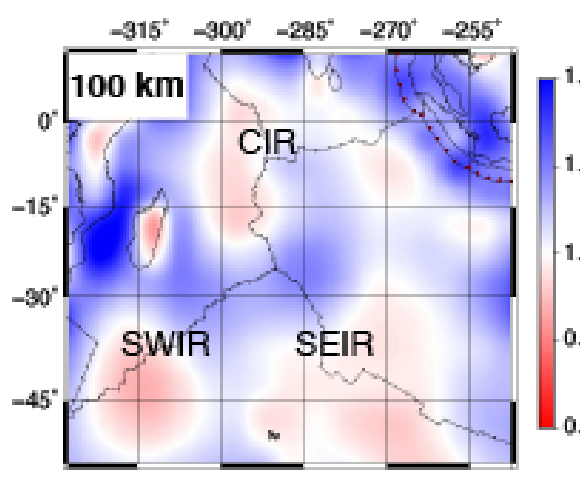

TB
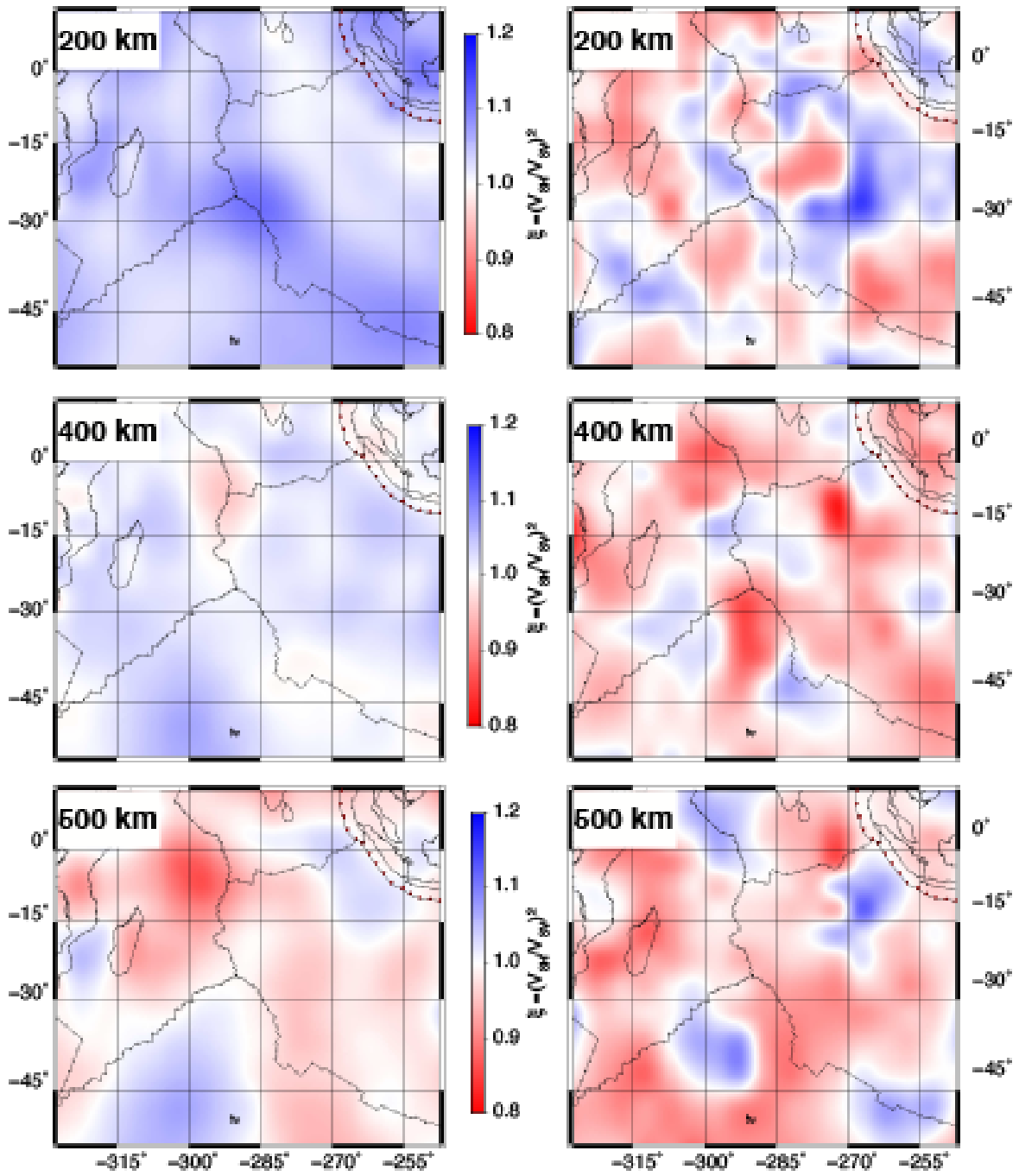

Figure 7. Shear-wave anisotropy estimated from the SVD inversion (left) and from the mean of the posterior distribution obtained with the non-linear TB inversion (right). The Central Indian Ridge (CIR), Southwestern Indian Ridge (SWIR), and Southeastern Indian Ridge (SEIR) branches are labeled in the top panels for reference. 
Africa and Madagascar and east of the CIR. At $500 \mathrm{~km}$, relative negative or positive velocity perturbations of $5 \%$ or more are very unlikely to exist. At those depths, positive anomalies of at least 1\% may exist near La Réunion Island and the SEIR, and negative anomalies of at least $1 \%$ may exist on the SWIR and east of the CIR.

Figs. 10 and 11 reveal that at $100 \mathrm{~km}$ depth, there is a high likelihood $(P \geq 0.75)$ for $V_{S H}>V_{S V}$ with strength of at least $5 \%$ underneath much of the Indian ocean. The exceptions are the RTJ, where the CIR, SEIR, and SWIR intersect, and the region around the Indonesian subduction zone where the likelihood of $\xi>1$ is low (Fig. 10). At the RTJ, the likelihood of $\xi<1$ is also weak (Fig. 11), suggesting the area may not have any radial anisotropy. A small pocket of likely $V_{S H}<V_{S V}$ with strength of at least $5 \%$ is also visible south of the Indonesian slab around $30^{\circ} \mathrm{S}, 105^{\circ} \mathrm{E}$. At $200 \mathrm{~km}$, the likelihood of anisotropy anomalies is more heterogeneous and we see both horizontally fast and vertically fast anomalies of about $1 \%$, indicating a likely reduction in the amplitude of the anisotropy with depth. There is also a pocket east of the RTJ with likely horizontally fast velocities with at least $5 \%$ anisotropy. At $400 \mathrm{~km}$ depth, the likelihood of $V_{S H}>V_{S V}$ decreases further and a few locations are characterized by a probability of 0.7 or greater of having $V_{S H}<V_{S V}$ with about $1 \%$ anisotropy $(\xi \simeq 0.99)$. At that depth, a zone of likely vertically fast velocities with at least $5 \%$ anisotropy is found south of the RTJ and in between the the CIR and Indonesian subduction zone. At $500 \mathrm{~km}$, we see that much of the region has likely $V_{S H}<V_{S V}$ with $0.95<\xi<0.99$.

\subsection{TB Inversions Synthetic Tests}

In order to test the robustness of the above results, we ran synthetic tests using some of the mean models displayed in Figs. 6 and 7 as input to calculate the synthetic data. The prior and the noise parameterization were defined in the same way as in the real data inversions. Fig. 12 shows the result of one such test. For $V_{S V}$ and the reconstructed $V_{S}$, we find that the posterior model distribution encompasses the input model and that the mean of this distribution is representative of the input model, especially above $400 \mathrm{~km}$ depth. At greater depths, the uncertainties increase. For $V_{S H}$, the peaks of the posterior distribution are offset compared to the input model, showing that the TB inversion tends to overestimate the model amplitudes. However, the mean model follows the input model at most depths except between $400 \mathrm{~km}$ and $550 \mathrm{~km}$ where rapid vertical changes in the input model are not accurately captured by the mean of the posterior distribution. This is likely due to limitations in the vertical resolution of the data. Additionally, the $50 \mathrm{~km}$ to $150 \mathrm{~km}$ depth range is characterized by multiple peaks in the posterior distribution that indicate trade-offs: the data cannot distinguish between 

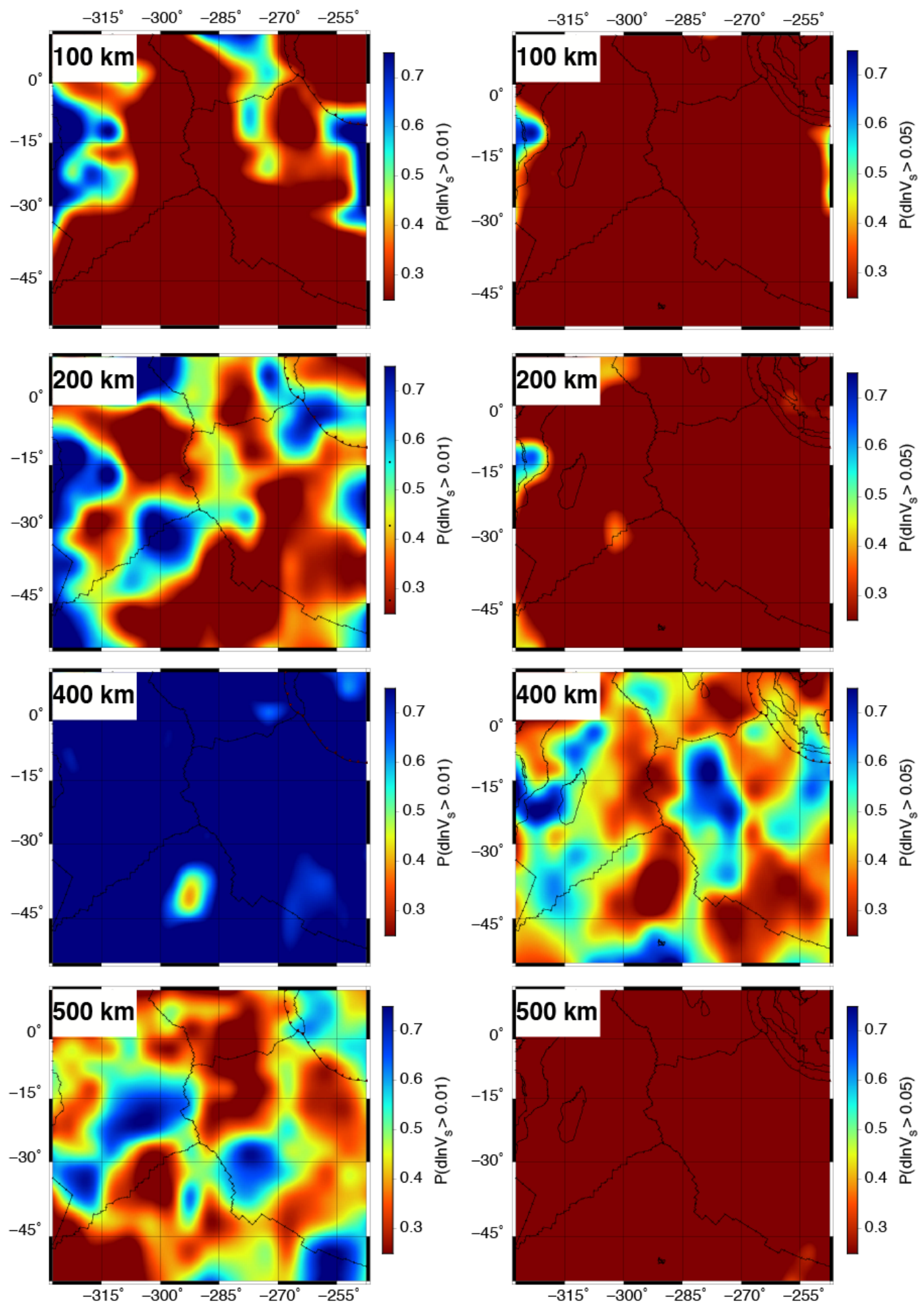

Figure 8. Probability of $d \ln V_{S}>1 \%$ (left) and $d \ln V_{S}>5 \%$ (right) at different depths. 

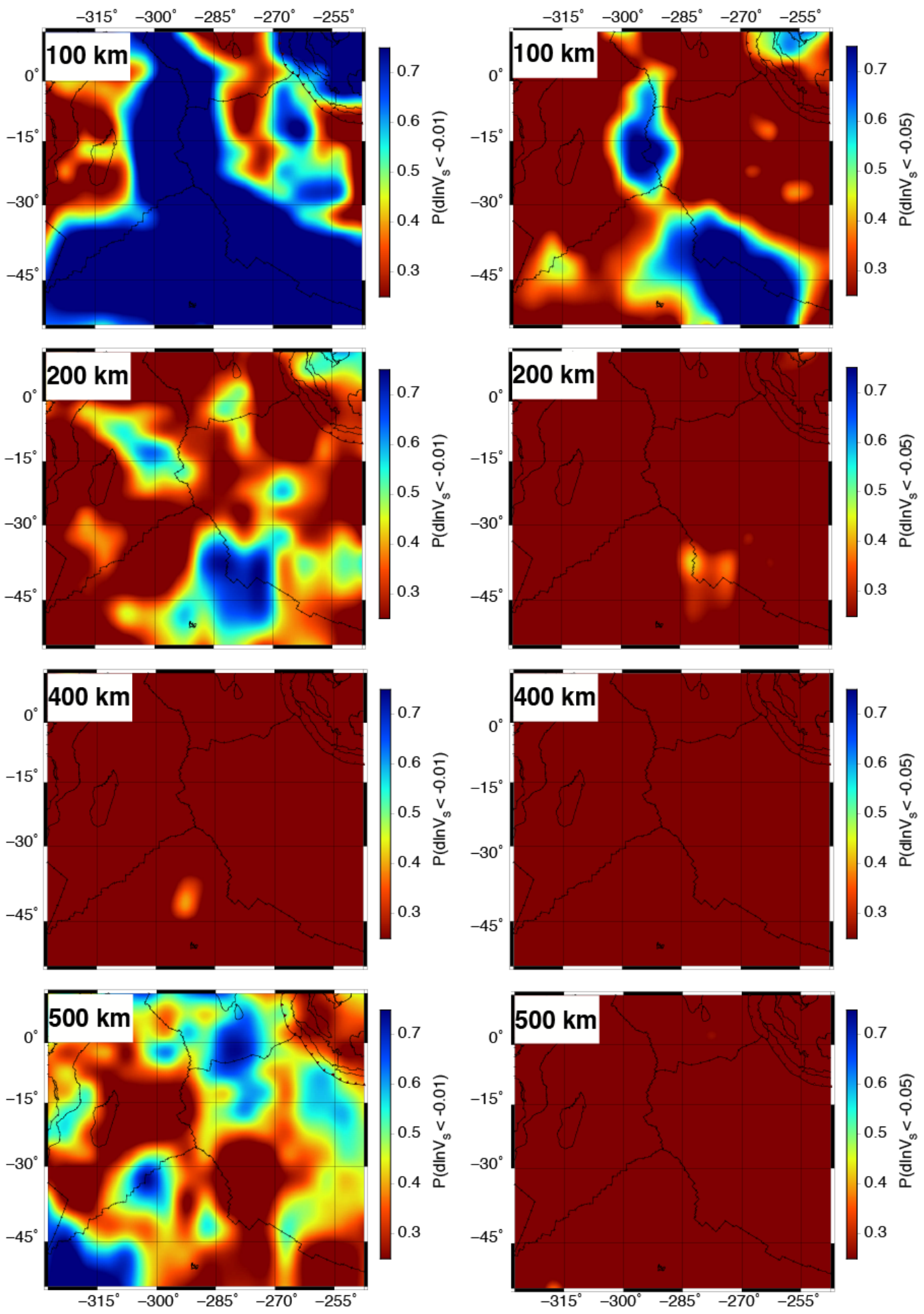

Figure 9. Probability of $d \ln V_{S}<-1 \%$ (left) and $d \ln V_{S}<-5 \%$ (right) at different depths. 

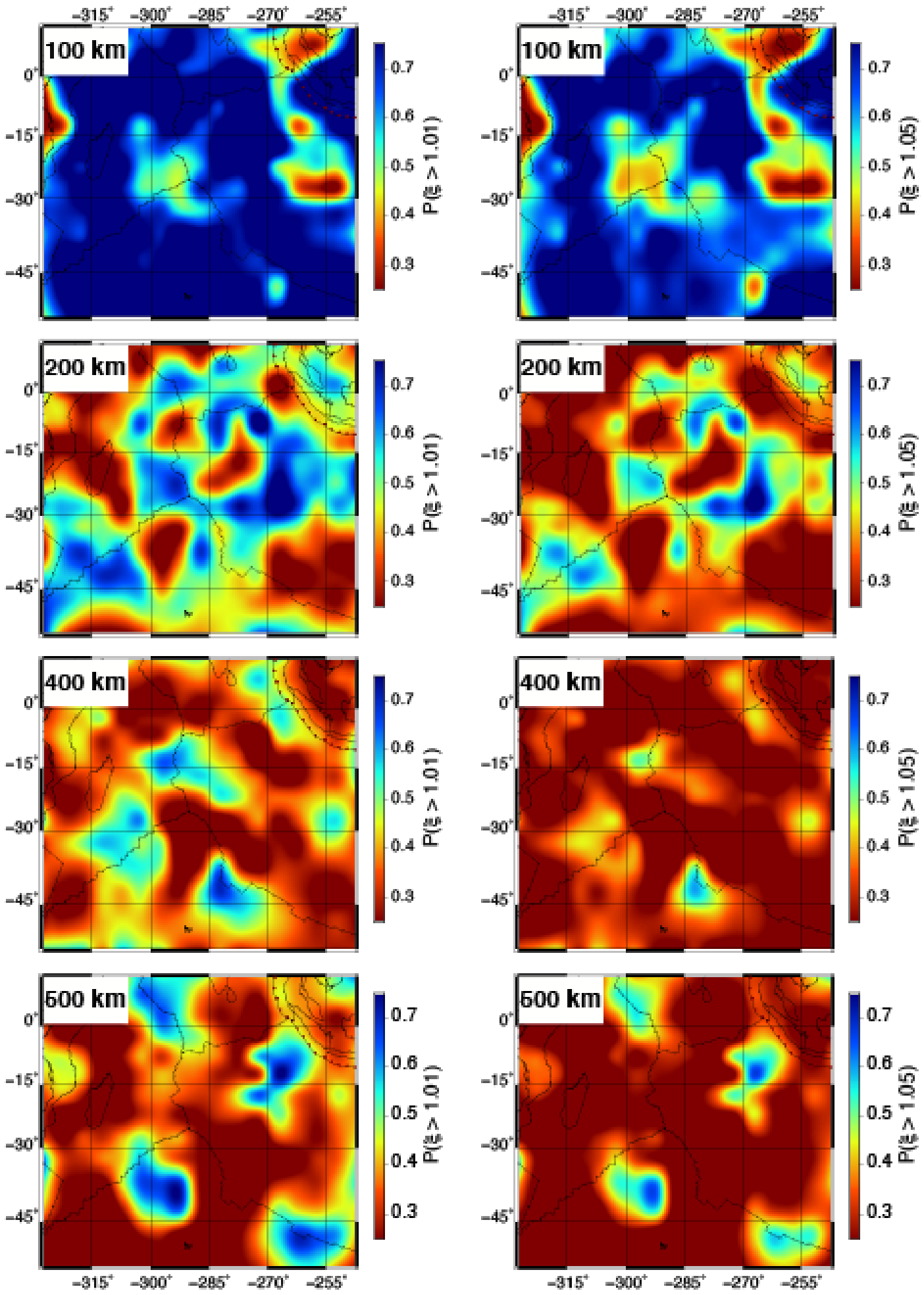

Figure 10. Probability of having $V_{S H}>V_{S V}$ (i.e., $\xi>1$ ) at different depths with at least $1 \%$ (left) and $5 \%$ (right) anisotropy. 

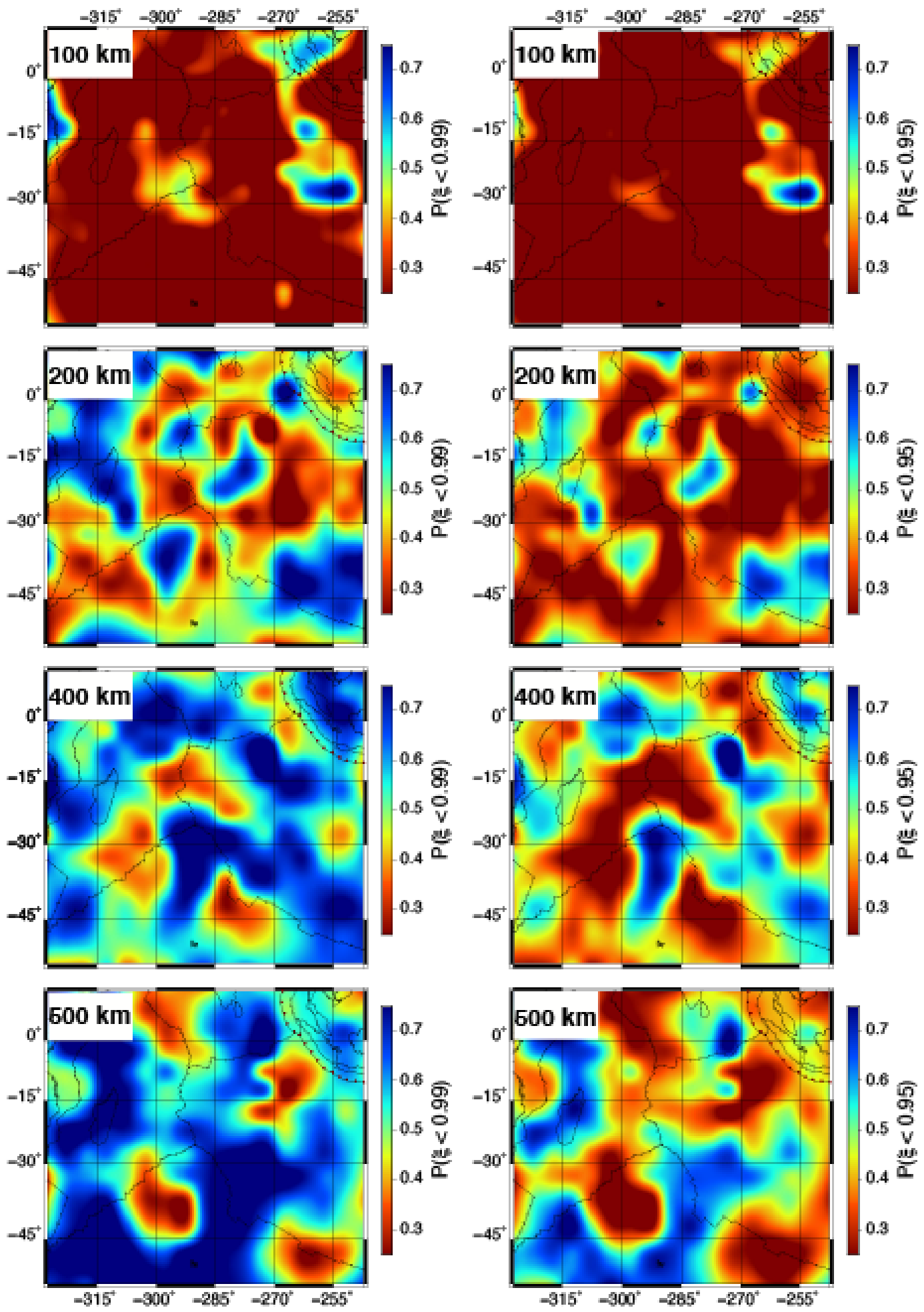

Figure 11. Probability of having $V_{S H}<V_{S V}$ (i.e., $\xi<1$ ) at different depths with at least $1 \%$ (left) and $5 \%$ (right) anisotropy. 
a high velocity zone above a low velocity layer and a constant velocity structure at these depths. Since $\xi$ is the square of the ratio of $V_{S H}$ to $V_{S V}$, these differences between input and mean model are amplified in the reconstructed $\xi$ profile. We see that the general trend of the posterior distribution roughly follows that of the input $\xi$ model, but the amplitudes of the input model and the mean or the peak of the posterior distribution differ. Nevertheless, it should be noted that the posterior $\xi$ distribution encompasses the input model, which highlights the importance of accounting for model uncertainties when interpreting results.

A question that may still arise is whether there could be leakage from isotropic to anisotropic structure. We thus ran another synthetic test with an isotropic input model below $200 \mathrm{~km}$ (Fig. 13). As in Fig. 12, we find a close match between the input model and the mean of the posterior for $V_{S V}$ and $V_{S}$ down to about $400 \mathrm{~km}$ depth. At greater depths, the posterior becomes wider, and the mean model oscillates around the input model. For $V_{S H}$, the same multimodal peaks found in Fig. 12 are visible between $50 \mathrm{~km}$ and $150 \mathrm{~km}$, and deviations are found between the input and mean posterior model around $200 \mathrm{~km}$, which marks the abrupt transition between anisotropy and isotropy in the input model. At larger depths, the mean $V_{S H}$ model follows the input model relatively well, though with larger uncertainties. For radial anisotropy, the mean model shows a strong $\xi<1$ near $200 \mathrm{~km}$ depth that peaks about $50 \mathrm{~km}$ deeper than the corresponding anomaly of the input model. At larger depths, the mean model oscillates around the isotropic input model, with peak anisotropy approaching $5 \%$ at some depths. If one were to interpret these results based on the mean anisotropy model alone, one would conclude that there is a risk of finding deep anisotropy with our method that is not constrained by the data. However, we note that the posterior $\xi$ distribution below $200 \mathrm{~km}$ is wide and allows a wide range of values of both $\xi>1$ and $\xi<1$ at these depths. This deep anisotropy found in the mean model is therefore not resolved, but this again highlights how essential it is to determine the posterior model uncertainties before interpreting the anisotropy signal.

In order to determine the level of confidence with which we can interpret the likelihood map shown in Figs. 10 and 11, we used the posterior $\xi$ distribution displayed in Fig. 13 to calculate the likelihood of finding $1 \%$ and $5 \%$ anisotropy for both $V_{S V}>V_{S H}$ and $V_{S H}>V_{S V}$. The results are shown in Fig. 14. We see a strong likelihood $(P=0.8)$ of finding $\xi<0.95$ between $200 \mathrm{~km}$ and $\sim 230 \mathrm{~km}$, where the input model is isotropic. We attribute this to the inability of the surface waves to recover a sharp velocity or anisotropy vertical change. Most noteworthy, however, is the fact that below $\sim 250 \mathrm{~km}$ depth, the probability that we might find an anisotropy signal not constrained by the data is generally less than 0.6 . This means 

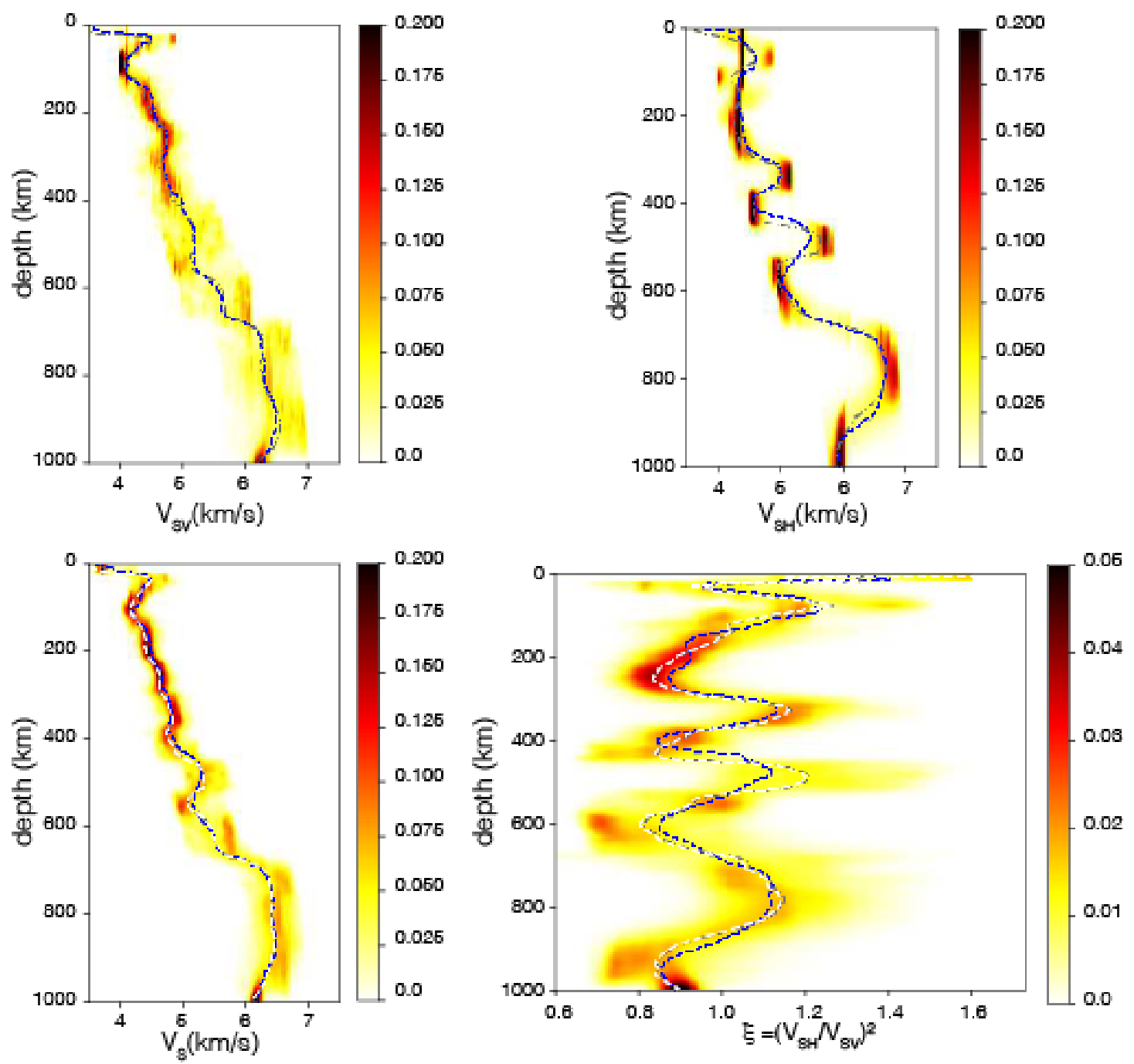

Figure 12. Example of synthetic test. Top: Posterior $V_{S V}$ and $V_{S H}$ distributions resulting from the separate Rayleigh and Love wave synthetic dispersion data; Bottom: corresponding $V_{S}$ and $\xi$ posterior distributions. The color scale represents the probability that a model parameter has a specific value at a given depth. The input model is shown as the dashed blue line. The dash-dotted grey line is the mean of the posterior distribution. The dashed white line in the $V_{S}$ and $\xi$ profiles calculated using the mean $V_{S V}$ and mean $V_{S H}$ results.

that, when discussing our results (Figs. 10 and 11), we can confidently conclude that the deep anisotropy signal in our models is constrained by the data as long as we only interpret anisotropy associated with a likelihood higher than 0.6. 
(A)
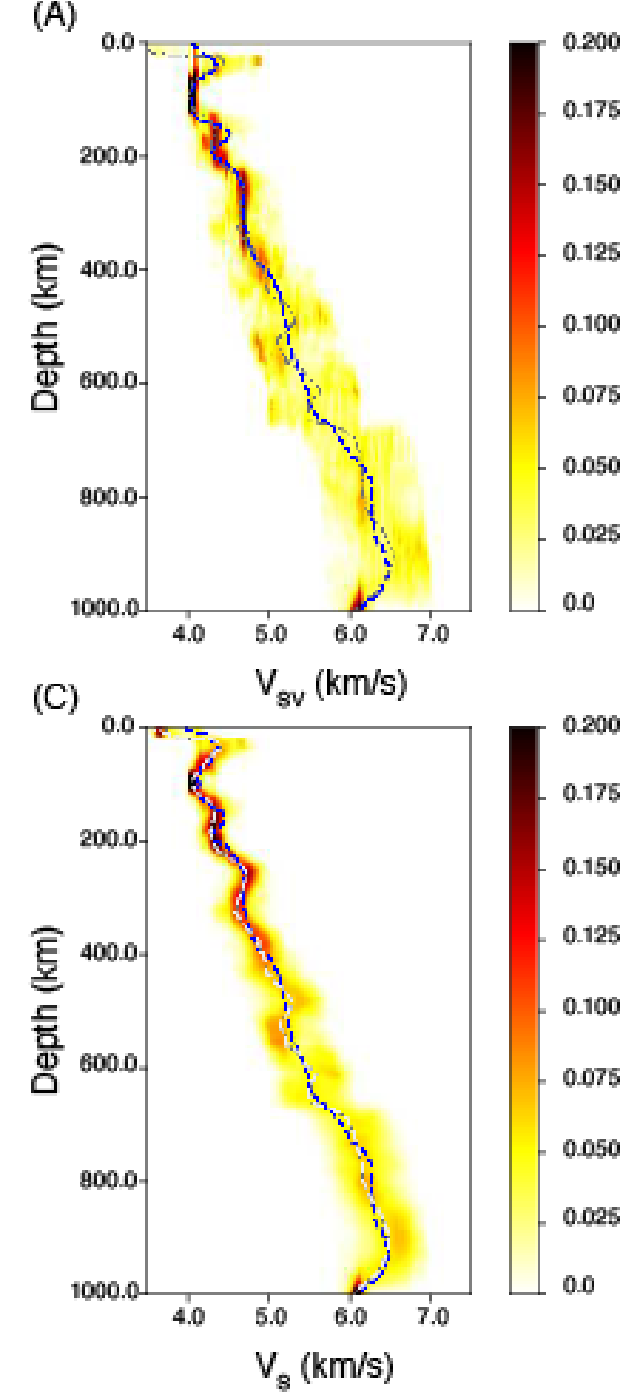

(B)
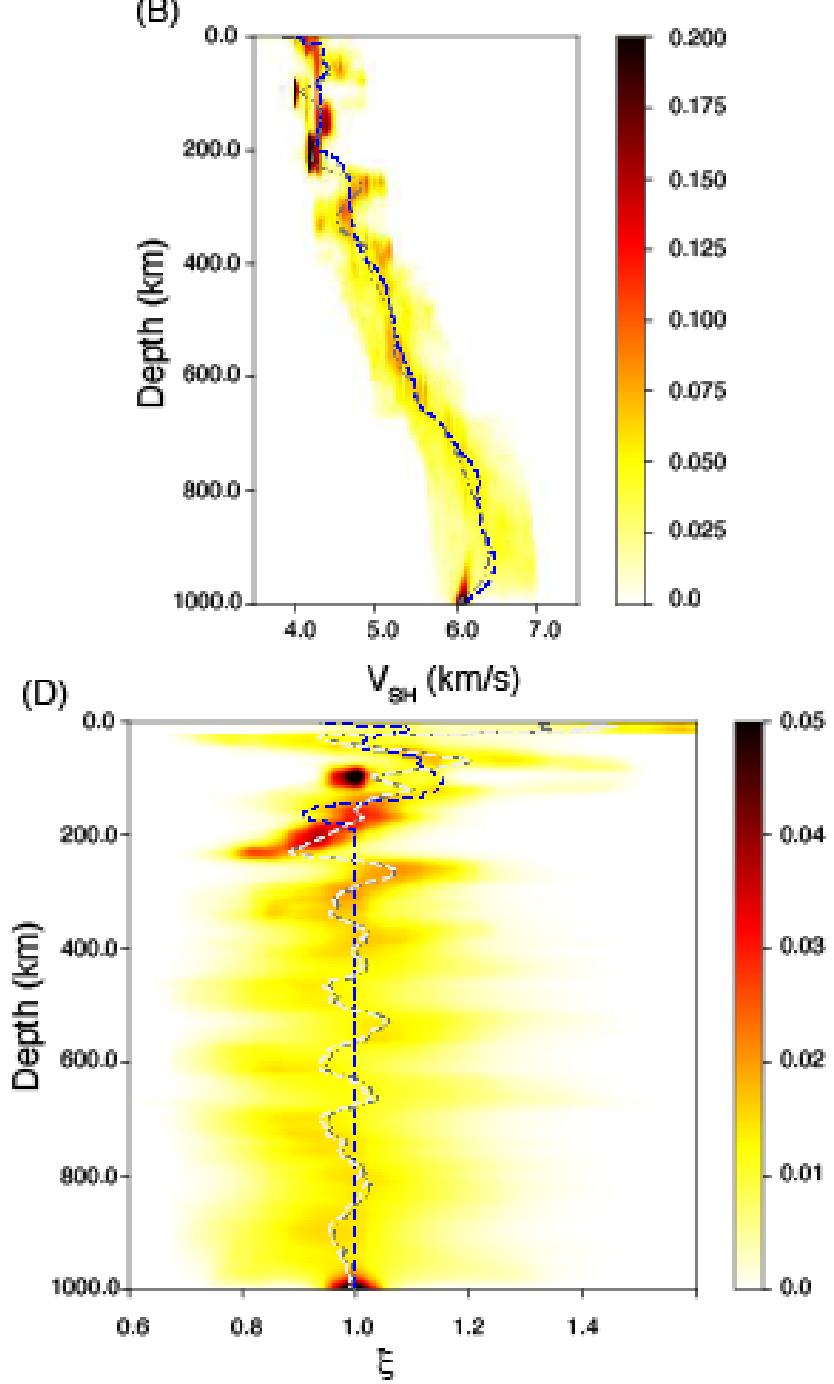

Figure 13. Synthetic test using an input model that is isotropic below $220 \mathrm{~km}$ depth. The color scale indicates the probability that the model parameter has a specific value at a given depth. The input model is shown as a dashed blue line. The dashed-dotted grey line is the mean model of the posterior distribution. The dashed white line in the $V_{S}$ and $\xi$ plots was obtained from the mean $V_{S V}$ and mean $V_{S H}$ results.

\subsection{Joint Group and Phase Velocity Inversion}

Some of the results presented above show multiple peaks in the posterior distributions, indicating that trade-offs exist between the different depth parameters (Fig. 4). In addition, it is clear that Love wave phase velocity data alone cannot resolve $V_{S H}$ as well as Rayleigh wave phase velocities can resolve $V_{S V}$, resulting in large uncertainties in $\xi$. Group velocity dispersion data are generally sensitive to shallower depths than phase velocities at the same periods 

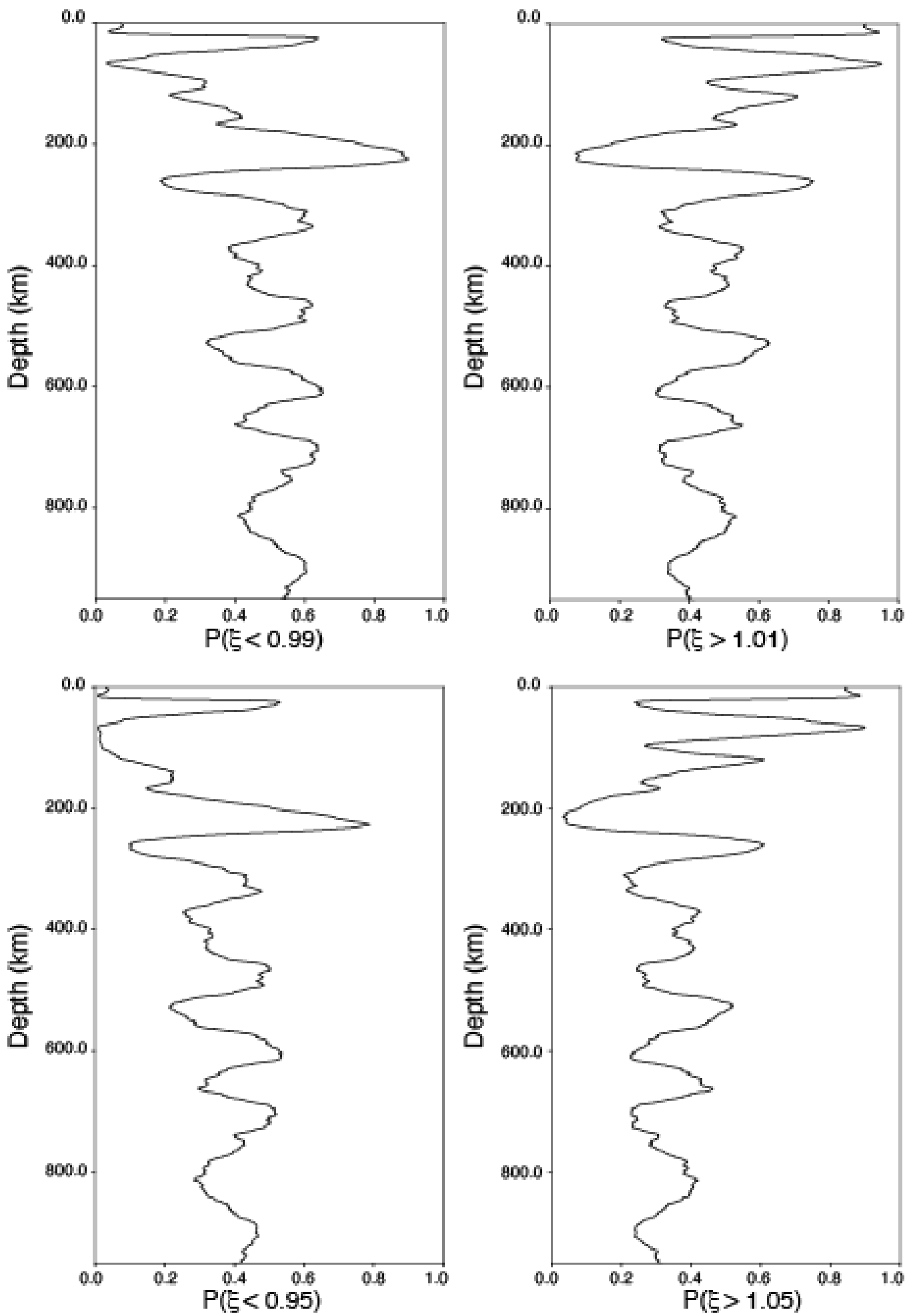

Figure 14. Depth profile of likelihood of $\xi$ anomalies relative to isotropy $(\xi=1)$ based on posterior distributions shown in Fig. 13. 

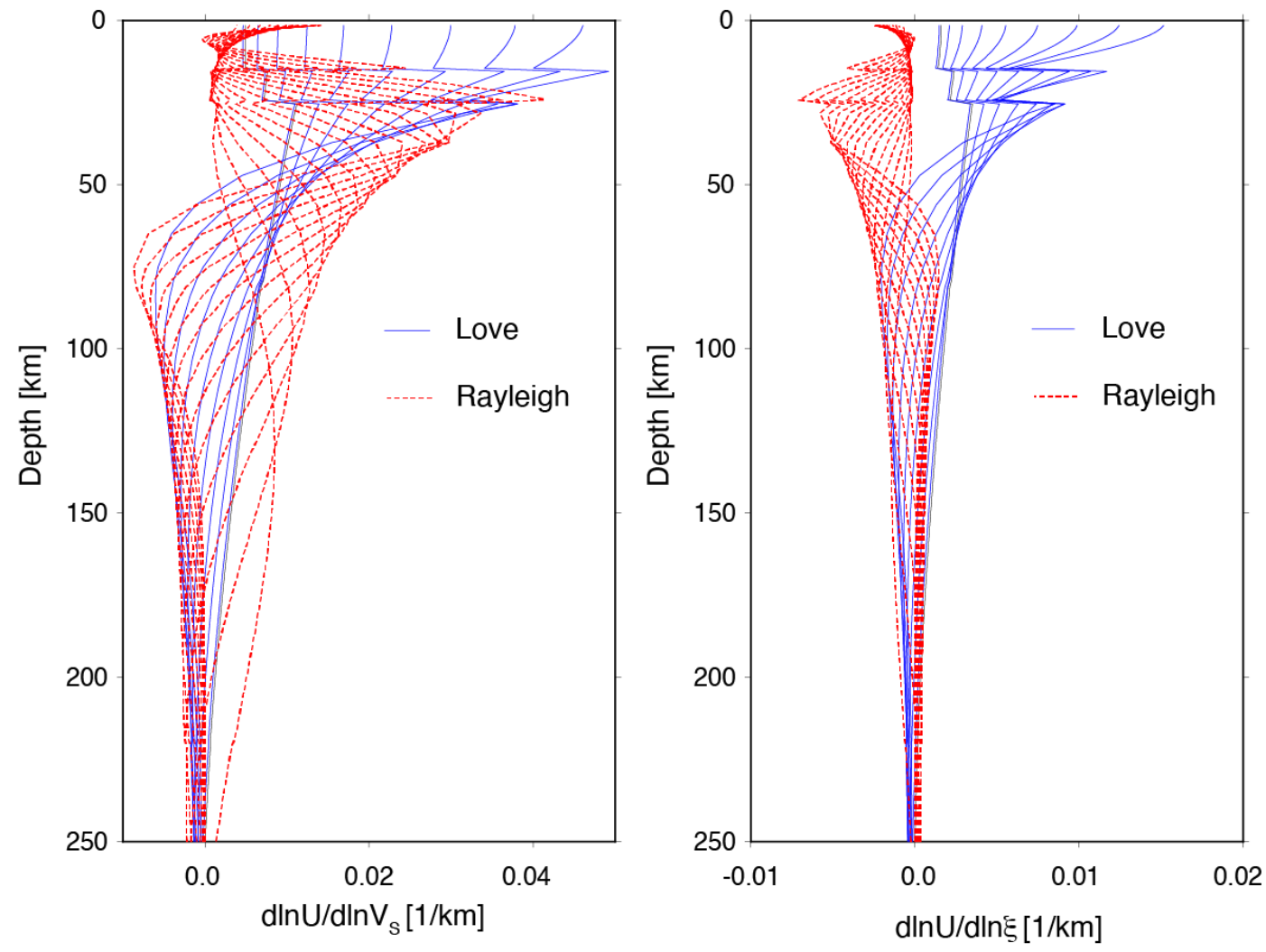

Figure 15. Fundamental mode group velocity sensitivity kernels for perturbations in $V_{S}$ (left) and $\xi$ (right) between $10 \mathrm{~s}$ and $30 \mathrm{~s}$ for Love waves and between $7.5 \mathrm{~s}$ and $40 \mathrm{~s}$ for Rayleigh waves.

(Fig. 15) and may therefore help break trade-offs between shallow and deep structure. In this section, we tested whether adding the group velocity data measured by Ma et al. (2014) could reduce trade-offs and help better constrain the deep anisotropy signal.

Since the group and phase velocity data came from two different studies, we included independent noise parameters for each data type in our inversions. Fig. S6 represents the posterior noise distributions. It shows that the Love wave data are generally noisier than the Rayleigh wave data and that the group velocity data also contain a larger amount of noise than the phase velocities. Fig. 16 compares the posterior model distributions for an inversion with both phase and group velocity measurements and an inversion with phase velocity measurements only. Both inversions use data at the same grid cell: $\left(3^{\circ} \mathrm{S}, 79^{\circ} \mathrm{E}\right)$. We find that the multimodal aspect of the $V_{S H}$, and to some extent $V_{S V}$, PPDFs at shallow depths is less pronounced when group velocities are included in the dataset. This leads to narrower distributions for $V_{S}$ and $\xi$ in the top $50 \mathrm{~km}-100 \mathrm{~km}$, showing that the addition of group velocities improves constraints on the shallow structure. The amplitude of the mean 

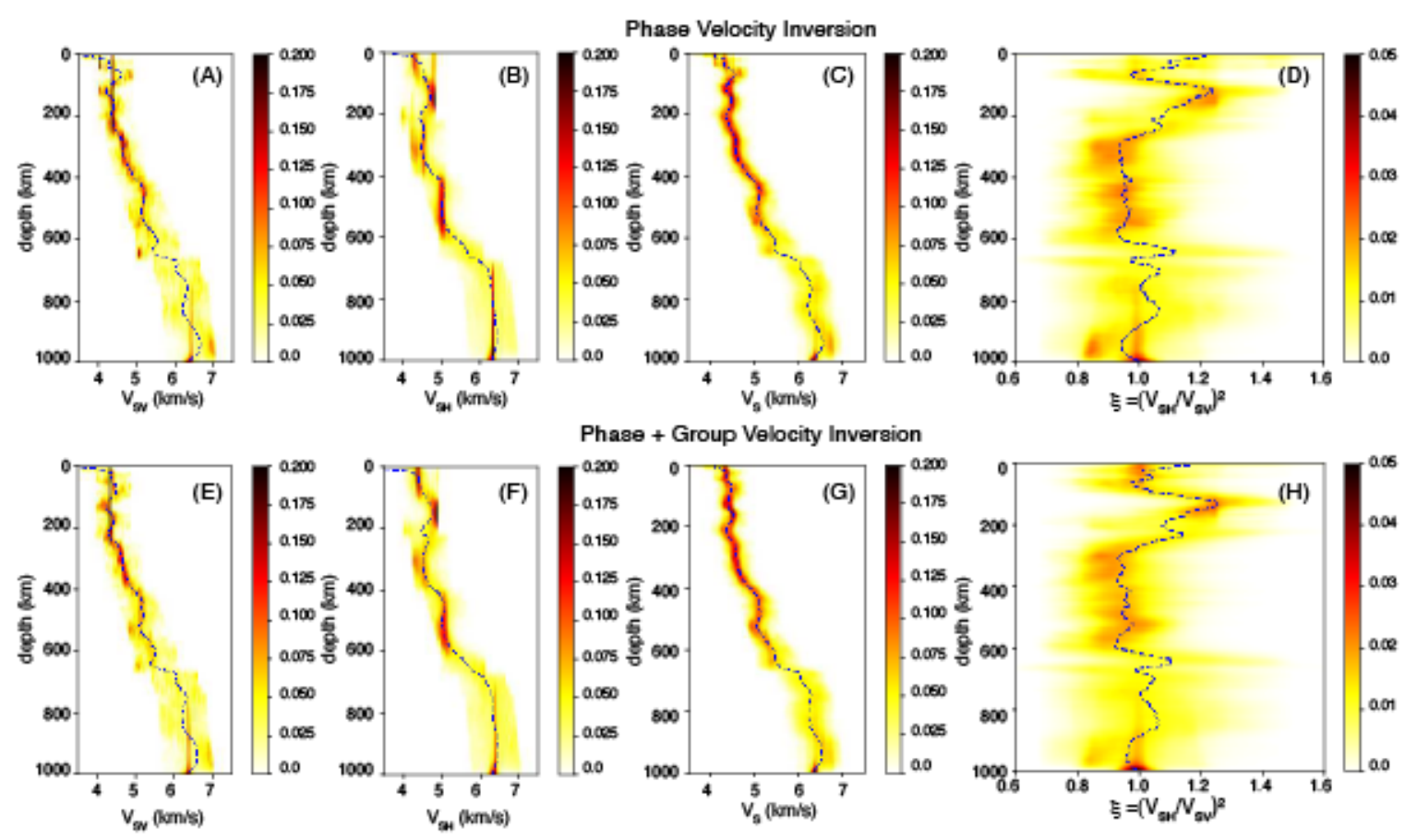

Phase + Group Velocity Inversion
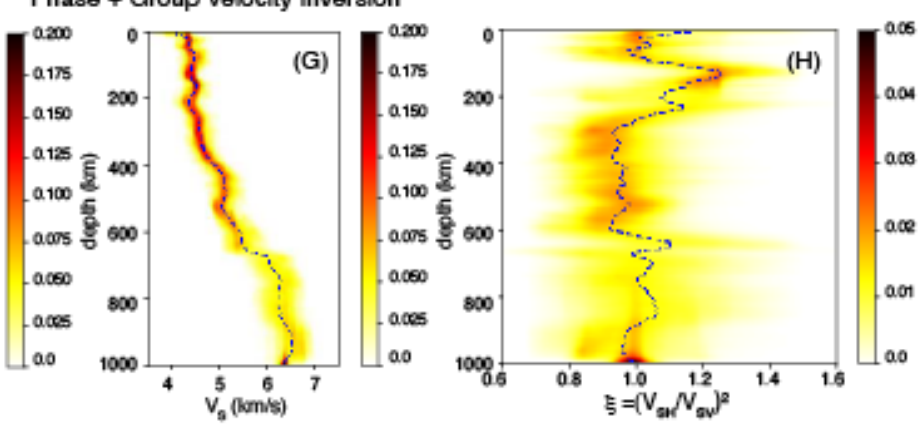

Figure 16. Comparison of inversion of phase velocity data only (top) and joint inversion of phase and group velocity data (bottom) at $3^{\circ} \mathrm{S}, 79^{\circ} \mathrm{E}$. Inversions were performed separately for $V_{S V}((\mathrm{~A})$ and $(\mathrm{E}))$ and for $V_{S H}((\mathrm{~B})$ and $(\mathrm{F}))$. Panels $(\mathrm{C})$ and $(\mathrm{G})$ represent the reconstructed posterior $V_{S}$ model distribution and (D) and (H) represent the reconstructed $\xi$ model distribution. The dashed lines correspond to the mean of the distributions.

anisotropy model is also found to be slightly lower in the top $50 \mathrm{~km}$ when group velocities are included but the general trend does not change significantly. Importantly, we find that the posterior distributions do not change significantly below $\sim 100 \mathrm{~km}$. This suggests that, although the group velocity data can help constrain the shallow structure better, it does not change the model resolution in the rest of the upper mantle and does not affect our interpretation of the results.

\section{DISCUSSION}

The surface wave tomography of Debayle \& Lévêque (1997) revealed that the lowest shear wave velocity anomalies at $100 \mathrm{~km}$ depth were located along the ridges, with the CIR and SEIR showing stronger anomalies than the SWIR. Low velocities along the CIR are also seen in the fundamental Rayleigh wave phase and group velocity study of Mazzullo et al. (2017). This is in agreement with our mean model (Fig. 6) and is confirmed by our likelihood estimates. Fig. 9 indeed shows that both the CIR and SEIR are likely to have $d \ln V_{S} \leq-0.05$ while 
the SWIR is characterize by a high likelihood of $d \ln V_{S} \leq-0.01$ but not $d \ln V_{S} \leq-0.05$. Interestingly, our results suggest that the low velocity anomalies seen under the CIR and SEIR persist down to $\sim 200 \mathrm{~km}$ as in Debayle \& Lévêque (1997), but that the SWIR has a shallower origin. Debayle \& Lévêque (1997) found that these anomalies largely disappear at around $200 \mathrm{~km}$. This is qualitatively consistent with our results, which show a decrease in the amplitude of the anomalies. At $400 \mathrm{~km}$, our results favor a positive velocity perturbation of at least $1 \%$ and likely less than $5 \%$ across the whole study area, which suggests a relatively cold mantle. This differs from several other studies (Ritsema et al. 2011; Kustowski et al. 2008; French \& Romanowicz 2014), though exceptions include the work of Panning \& Romanowicz (2006b); Panning et al. (2010) and Auer et al. (2014), which displayed $d \ln V_{S}>0$ along ridges at similar depths. In the MTZ, our models present weak positive and negative velocity anomalies of about $1 \%$, qualitatively similar to other studies.

As in Visser et al. (2008a), we find a high likelihood $(P \geq 0.75)$ of $V_{S H}>V_{S V}$ at $100 \mathrm{~km}$ depth throughout most of the study region. This is consistent with previously published radial anisotropy models of our study area (Lévèque et al. 1998) as well as in other regions and globally (Gung et al. 2003b; Ekström \& Dziewonski 1998; Panning \& Romanowicz 2006b; Kustowski et al. 2008; Panning et al. 2010; Auer et al. 2014; French \& Romanowicz 2014; Chang et al. 2015) and is often interpreted as the signature of horizontal asthenospheric motion. At $200 \mathrm{~km}$, our models present likely $(P \geq 0.75) V_{S V}>V_{S H}$ and $V_{S H}>V_{S V}$ anomalies with 1-5\% anisotropy scattered across the region, which differs somewhat from Visser et al. (2008a) who found a generally low likelihood of $V_{S H}>V_{S V}$ at that depth. No clear anisotropy pattern or relation to geological features emerges from our results below $100 \mathrm{~km}$ depth except for a vertically fast signal at $200 \mathrm{~km}$ along the Indonesian subduction zone (Fig. 7), though it is likely to be of small amplitude (Fig. 11). Contrary to the global models of Gung et al. (2003b); Panning \& Romanowicz (2006b) and Zhou et al. (2006), we do not see any clear anisotropy signal $V_{S V}>V_{S H}$ underneath ocean ridges below $100 \mathrm{~km}$. Overall, the tendency of the higher mode data to favor $V_{S V}>V_{S H}$ in the deep mantle, including the MTZ, found by Visser et al. (2008a) is similar to what we found, though their results seem to suggest a more ubiquitous $\xi<1$ signal than ours. Other models have previously shown small, laterally variable radial anisotropy in the MTZ (Auer et al. 2014; Moulik \& Ekström 2014) though they do not necessarily share the same patterns. Assuming anisotropy at those depths is due to the strain-induced lattice preferred orientation of intrinsically anisotropic minerals, our results suggest that the higher mode data favor a generally vertical flow signal below $200 \mathrm{~km}$ down to the MTZ. It should, however, be noted that the relationship between fast 
seismic direction and mantle deformation is not straightforward at those depths. Unlike for the shallow mantle, the anisotropy fast axis may not be a good proxy for flow direction in this part of the mantle as deformation could occur with a different slip system than at shallower depths (Mainprice et al. 2005). As pointed by Yuan \& Beghein (2013), recrystallization or a change in the slip system of the olivine structure during phase changes at $410 \mathrm{~km}$ depth could also affect the relation between observed seismic anisotropy and flow direction.

\section{CONCLUSION}

We developed a non-linear inversion method based on Markov Chain Monte Carlo within a transdismensional Bayesian framework to constrain Earth's interior structure with fundamental and higher mode surface wave dispersion measurements. Besides the ability to solve the non-linear problem, the advantages of this approach include the fact that the choice of the depth parametrization is iteratively driven by the data. The results are therefore not influenced by an arbitrary prior choice of the number of model parameters. Another advantage lies in the ability to invert the noise level jointly with the model parameters, which reduces the risk of mapping unknown data noise into the models. In addition, the use of a Monte Carlo approach enables us to sample all possible models and to represent the ensemble of solutions with a distribution of parameters instead of choosing one model among many possibilities. This allowed us to account for model uncertainties by calculating a likelihood for every parameter of interest.

We applied our technique on a subset of the global phase velocity maps obtained by Visser et al. (2008b) for Love and Rayleigh waves up to the fifth and sixth overtone, respectively. We focused on the Indian ocean and determined how well these data can constrain radial anisotropy in the deep upper mantle and transition zone. We found that correcting the measurements for the effect of perturbations in the depth of the MTZ boundaries did not impact the resulting models significantly, despite the higher mode data having sensitivity to these depths. We also demonstrated that separate Love and Rayleigh wave inversions produced results equivalent within model uncertainties to those of joint inversions, as long as the depth parameterizations for $V_{S V}$ and $V_{S H}$ are allowed to differ in the joint inversions.

The velocity models resulting from the mean of the posterior model distribution were in general agreement with results from linearized inversions, though with stronger negative anomalies along ridges in the upper $100 \mathrm{~km}-200 \mathrm{~km}$. Stronger deviations between the models were found at $400 \mathrm{~km}$ depth, but they appeared to be in better agreement in the MTZ. This suggests that using a fully non-linear TB method to constrain shear-wave velocities with 
higher mode surface waves may not result in significant changes in the model compared to regularized inverse methods. Our results show, however, that using such as a technique and quatifying model uncertainties is essential when discussing models of radial anisotropy. Large discrepancies were indeed found between the two methods for $\xi$, even at $100 \mathrm{~km}$ depth where the data sensitivity was high, and model uncertainties were larger below $\sim 300 \mathrm{~km}$. Despite these large posterior model uncertainties, we were able to calculate the likelihood of anisotropy at different depths and determine how well resolved deep anisotropy signal is. Overall, while the dominant signal at $100 \mathrm{~km}$ is $V_{S H}>V_{S V}$ as expected for seismic anisotropy resulting from horizontal mantle motion, we found likely lateral variations in $\xi$ at greater depths, with a tendency for the data to favor a small amount of anisotropy with $V_{S V}>V_{S H}$ across most of the region in the deep upper mantle and MTZ. The synthetic tests we performed also demonstrated that the anisotropy signal found at these depths is resolved by the higher mode data and does not result from leakage. We also found that the inclusion of group velocity dispersion data may help reduce parameter trade-offs in the top $100 \mathrm{~km}$ of the mantle but does not improve the model resolution in the deep upper mantle or MTZ.

For our TB inversions, we chose a prior that would not significantly influence the results. Thus, the posterior distribution is mostly a function of the likelihood of a model given the misfit of the predicted data and the observations. This results in models that can explain the data used, but may not necessarily be in agreement with other datasets. This is reflected in some of the very large deviations of $\xi$ from 1 (isotropy), with the posterior distributions sometimes including anisotropy of $25 \%$ or more. Additional information could improve constraints on model parameters, guiding the posterior distributions to more realistic values. This could take the form of complementary types of data that reduce trade-offs from the current data set. This could also take the form of a narrower prior, which could be based on mineral physics. This will be implemented in future work.

\section{ACKNOWLEDGMENTS}

This project was funded by NSF EAR grant \#1446978. The data used in this study were downloaded from http://www.geo.uu.nl/ jeannot/JTweb/downloads.html for the phase velocity maps, and from https://igppweb.ucsd.edu/ gabi/litho1.0.html\#surfcodes for the group velocity data. 


\section{REFERENCES}

Anderson, D. L., 1962. Love wave dispersion in heterogeneous anisotropic media, Geophysics, 27, $445-454$.

Auer, L., Boschi, L., Becker, T. W., Nissen-Meyer, T., \& Giardini, D., 2014. Savani: A variable resolution whole-mantle model of anisotropic shear velocity variations based on multiple data sets, J. geophys. Res. Solid Earth, 119, 3006-3034.

Babuska, V. \& Cara, M., 1991. Seismic Anisotropy in the Earth, vol. 10, Springer Science \& Business Media.

Bayes, T., 1763. An Essay Towards Solving a Problem in the Doctrine of Chances, Philos. Trans. R. Soc., 53, $370-418$.

Becker, T. W., Kellogg, J. B., Ekström, G., \& O'Connell, R. J., 2003. Comparison of azimuthal seismic anisotropy from surface waves and finite strain from global mantle-circulation models, Geophys. $J$ Int., 155, 696-714.

Beghein, C. \& Trampert, J., 2004. Probability density functions for radial anisotropy: implications for the upper $1200 \mathrm{~km}$ of the mantle, Earth Planet. Sci. Lett., 217, 151-162.

Beghein, C. \& Trampert, J., 2006. Radial anisotropy in seismic reference models of the mantle, $J$. geophys. Res., 111(B02303).

Beghein, C., Yuan, K., Schmerr, N., \& Xing, Z., 2014. Changes in Seismic Anisotropy Shed Light on the Nature of the Gutenberg Discontinuity, Science, 343, 1237-1240.

Bodin, T., Sambridge, M., Tkalčić, H., Arroucau, P., Gallagher, K., \& Rawlinson, N., 2012. Transdimensional inversion of receiver functions and surface wave dispersion, J. geophys. Res. Solid Earth, $117(\mathrm{~B} 2)$.

Bodin, T., Leiva, J., Romanowicz, B., Maupin, V., \& Yuan, H., 2016. Imaging anisotropic layering with bayesian inversion of multiple data types, Geophys. J Int., 206, 605-629.

Burgos, G., Montagner, J.-P., Beucler, E., Capdeville, Y., Mocquet, A., \& Drilleau, M., 2014. Oceanic lithosphere-asthenosphere boundary from surface wave dispersion data, J. geophys. Res. Solid Earth, 119, 1079-1093.

Chang, S.-J., Ferreira, A. M. G., Ritsema, J., Heijst, H. J. v., \& Woodhouse, J. H., 2015. Joint inversion for global isotropic and radially anisotropic mantle structure including crustal thickness perturbations, J. geophys. Res. Solid Earth, 120, 4278-4300.

Debayle, E. \& Lévêque, J. J., 1997. Upper mantle heterogeneities in the Indian Ocean from waveform inversion, Geophys. Res. Lett., 24, 245-248.

Debayle, E. \& Ricard, Y., 2013. Seismic observations of large-scale deformation at the bottom of fast-moving plates, Earth Planet. Sci. Lett., 376, 165-177.

Dziewonski, A. M. \& Anderson, D. L., 1981. Premlinary reference Earth model, Phys. Earth Planet. Inter., 25, 297-356.

Ekström, G. \& Dziewonski, A. M., 1998. The unique anisotropy of the Pacific upper mantle, Nature, 
394, 168-172.

Faccenda, M., Burlini, L., Gerya, T. V., \& Mainprice, D., 2008. Fault-induced seismic anisotropy by hydration in subducting oceanic plates, Nature, 455(7216), 1097-1100.

Ferreira, A. M. G., Faccenda, M., Sturgeon, W., Chang, S.-J., \& Schardong, L., 2019. Ubiquitous lower-mantle anisotropy beneath subduction zones, Nat. Geosci., 12, 301-306.

French, S. W. \& Romanowicz, B., 2014. Whole-mantle radially anisotropic shear velocity structure from spectral-element waveform tomography, Geophys. J Int., 199, 1303 - 1327.

Gallagher, K., Charvin, K., Nielsen, S., Sambridge, M., \& Stephenson, J., 2009. Markov chain monte carlo (mcmc) sampling methods to determine optimal models, model resolution and model choice for earth science problems, Mar. Pet. Geol., 26, 525-535.

Gao, C. \& Lekić, V., 2018. Consequences of parameterization choices in surface wave inversion: Insights from transdimensional Bayesian methods, Geophys. J Int..

Green, P. J., 1995. Reversible jump markov chain monte carlo computation and bayesian model determination, Biometrika, 82, 711-732.

Green, P. J., 2003. Trans-dimensional mcmc, in Highly Structured Stochastic Systems, chap. 6, pp. 179-196, eds Green, P. J., Hjort, N., \& Richardson, S., Oxford Statistical Science Series.

Gung, Y., Panning, M., \& Romanowicz, B., 2003a. Global anisotropy and thickness of continents, Nature, 422, 707-711.

Gung, Y., Panning, M. P., \& Romanowicz, B., 2003b. Global anisotropy and the thickness of continents, Nature, 422(6933), $707-711$.

Huang, Q., Schmerr, N., Waszek, L., \& Beghein, C., 2019. Constraints on Seismic Anisotropy in the Mantle Transition Zone From Long-Period SS Precursors, J. geophys. Res. Solid Earth, 124, 6779-6800.

Jackson, D. D., 1972. Interpretation of Inaccurate, Insufficient and Inconsistent Data, Geophys. J. R. Astron. Soc., 28(2), 97-109.

Karato, S.-I. \& Li, P., 1992. Diffusion Creep in Perovskite: Implications for the Rheology of the Lower Mantle, Science, 255, 1238-1240.

Kendall, J.-M. \& Silver, P. G., 1998. Investigating Causes of D" Anisotropy, in The Core-Mantle Boundary Region, vol. 18 of Geodynamics, pp. 97-116, American Geophysical Union.

Kustowski, B., Ekström, G., \& Dziewonski, A. M., 2008. Anisotropic shear-wave velocity structure of the earth's mantle: A global model, J. geophys. Res., 113, B06306.

Lanczos, C., 1961. Linear Differential Operators, D. Van Nostrand Reinhold Co.

Laske, G., Masters, G., Ma, Z., \& Pasyanos, M., 2013. Update on CRUST1.0 - A 1-degree Global Model of Earth's Crust, Geophys. Res. Abstracts, 15, Abstract EGU2013-2658.

Lynner, C. \& Long, M. D., 2014. Lowermost mantle anisotropy and deformation along the boundary of the African LLSVP, Geophys. Res. Lett., 41, 3447-3454.

Lynner, C. \& Long, M. D., 2015. Heterogeneous seismic anisotropy in the transition zone and upper- 
most lower mantle: evidence from South America, Izu-Bonin and Japan, gji, 201, 1545-1552.

Lévèque, J., Debayle, E., \& Maupin, V., 1998. Anisotropy in the Indian Ocean upper mantle from Rayleigh- and Love-waveform inversion, Geophys. J Int., 133, 529 - 540.

Ma, Z., Masters, G., Laske, G., \& Pasyanos, M., 2014. A comprehensive dispersion model of surface wave phase and group velocity for the globe, Geophys. J Int., 199, 113-135.

Mainprice, D., Tommasi, A., Couvy, H., Cordier, P., \& Frost, D. J., 2005. Pressure sensitivity of olivine slip systems and seismic anisotropy of Earth's upper mantle, Nature, 433(7027), 731-733.

Masters, G., Woodhouse, J. H., \& Freenan, G., 2011. Mineos.

Matsu'Ura, M. \& Hirata, N., 1982. Generalized least-squares solutions to quasi-linear inverse problems with a priori information., J. Phys. Earth, 30(6), 451-468.

Mazzullo, A., Stutzmann, E., Montagner, J.-P., Kiselev, S., Maurya, S., Barruol, G., \& Sigloch, K., 2017. Anisotropic Tomography Around La Réunion Island From Rayleigh Waves, J. geophys. Res. Solid Earth, 122, 9132-9148.

Meier, U., Trampert, J., \& Curtis, A., 2009. Global variations of temperature and water content in the mantle transition zone from higher mode surface waves, Earth Planet. Sci. Lett., 282, 91 - 101.

Montagner, J.-P., 1986. Regional three-dimensional structures using long-period surface waves, Ann. Geophys, 4, 283-294.

Montagner, J.-P. \& Anderson, D. L., 1989. Petrological Constraints on Seismic Anisotropy, Phys. Earth Planet. Inter., 54, 82-105.

Montagner, J.-P. \& Tanimoto, T., 1991. Global upper mantle tomography of seismic velocities and anisotropies, J. geophys. Res., 96(B12), 20337-20351.

Moulik, P. \& Ekström, G., 2014. An anisotropic shear velocity model of the Earth's mantle using normal modes, body waves, surface waves and long-period waveforms, Geophys. J Int., 199, 17131738 .

Panning, M. \& Romanowicz, B., 2006a. A three-dimensional radially anisotropic model of shear velocity in the whole mantle, Geophysical Journal International, 167(1), 361-379.

Panning, M., Lekić, V., Manga, M., Cammarano, F., \& Romanowicz, B., 2006. Long-period seismology on europa: 2. predicted seismic response, J. geophys. Res., 111, E12008.

Panning, M. P. \& Romanowicz, B., 2006b. A three-dimensional radially anisotropic model of shear velocity in the whole mantle, Geophys. J. R. Astron. Soc., 167, $361-379$.

Panning, M. P., Lekić, V., \& Romanowicz, B., 2010. Importance of crustal corrections in the development of a new global model of radial anisotropy, J. geophys. Res., 115, B12325.

Ritsema, J., Deuss, A., Heijst, H.-J. v., \& Woodhouse, J. H., 2011. S40RTS: a degree-40 shear-velocity model for the mantle from new Rayleigh wave dispersion, teleseismic traveltime and normal-mode splitting function measurements, Geophys. J. R. Astron. Soc., 184, $1223-1236$.

Sakamaki, T., Suzuki, A., Ohtani, E., Terasaki, H., Urakawa, S., Katayama, Y., Funakoshi, K.-i., Wang, Y., Hernlund, J. W., \& Ballmer, M. D., 2013. Ponded melt at the boundary between the 
lithosphere and asthenosphere, Nat. Geosci., 6, 1041-1044.

Sambridge, M., Gallagher, K., Jackson, A., \& Rickwood, P., 2005. Trans-dimensional inverse problems, model comparison and the evidence, Geophs. J. Int., 167, 528-542.

Schaeffer, A., Lebedev, S., \& Becker, T., 2016. Azimuthal seismic anisotropy in the Earth's upper mantle and the thickness of tectonic plates, Geophys. J Int., 207, 901-933.

Shapiro, N. M. \& Ritzwoller, M. H., 2002. Monte-Carlo inversion for a global shear-velocity model of the crust and upper mantle, Geophys. J Int., 151, 88-105.

Smith, D. B., Ritzwoller, M. H., \& Shapiro, N. M., 2004. Stratification of anisotropy in the Pacific upper mantle, J. Geophys. Res., 109.

Snieder, R. \& Trampert, J., 2000. Linear and Nonlinear Inverse Problems, in Geomatic Method for the Analysis of Data in the Earth Sciences, pp. 93-164, eds Dermanis, A., Grün, A., \& Sansò, F., Springer Berlin Heidelberg, Berlin, Heidelberg.

Tarantola, A., 2005. Inverse problem theory and methods for model parameter estimation, Society for Industrial and Applied Mathematics.

Trampert, J., 1998. Global seismic tomography: the inverse problem and beyond, Inverse Probl., 14, 371-385.

Visser, K., Trampert, J., Lebedev, S., \& Kennett, B., 2008a. Probability of radial anisotropy in the deep mantle, Earth Planet. Sci. Lett., 270, 241-250.

Visser, K., Trampert, J., \& Kennett, B. L. N., 2008b. Global anisotropic phase velocity maps for higher mode Love and Rayleigh waves, Geophys. J Int., 172, 1016-1032.

Wiggins, R. A., 1972. The general linear inverse problem: Implication of surface waves and free oscillations for Earth structure, Rev. Geophys., 10, 251-285.

Woodhouse, J. H. \& Dahlen, F. A., 1978. The effect of a general aspherical perturbation on the free oscillations of the Earth, Geophys. J. R. Astron. Soc., 53, 335-354.

Xing, Z. \& Beghein, C., 2015. A Bayesian approach to assess the importance of crustal corrections in global anisotropic surface wave tomography, Geophys. J Int., 203(3), 1832-1846.

Yuan, H. \& Romanowicz, B., 2010. Lithospheric layering in the North American craton, Nature, 466, 1063-1068.

Yuan, K. \& Beghein, C., 2013. Seismic anisotropy changes across upper mantle phase transitions, Earth and Planetary Science Letters, 374, 132-144.

Yuan, K. \& Beghein, C., 2014. Three-dimensional variations in Love and Rayleigh wave azimuthal anisotropy for the upper $800 \mathrm{~km}$ of the mantle, J. geophys. Res. Solid Earth, 119(4), 3232-3255.

Zhou, Y., Nolet, G., Dahlen, F. A., \& Laske, G., 2006. Global upper-mantle structure from finitefrequency surface-wave tomography, J. geophys. Res., 111, B04304. 